\title{
Chemistry During the Gas-rich Stage of Planet Formation
}

\author{
Edwin A. Bergin and L. Ilsedore Cleeves
}

\begin{abstract}
In this chapter we outline some of the basic understanding of the chemistry that accompanies planet formation. We discuss the basic physical environment which dictates the dominant chemical kinetic pathways for molecule formation. We focus on three zones from both observational and theoretical perspectives: (1) the planet forming midplane and ice/vapor transition zones (snow-lines), (2) the warm disk surface that is shielded from radiation, which can be readily accessed by todays observational facilities, and (3) the surface photodissociation layers where stellar radiation dominates. We end with a discussion of how chemistry influences planet formation along with how to probe the link between formation and ultimate atmospheric composition for gas giants and terrestrial worlds.
\end{abstract}

\section{Introduction}

Today with thousands of planets detected we are embarking on a new era of discovery. Front and center will be the telescope time-consuming task of probing the chemical composition of exoplanetary atmospheres. At the same time, the Atacama Large Millimeter Array (ALMA) has begun operation which has provided the first AU-scale resolved images of planet-forming disks. This has led to a revolution in our understanding of the beginnings of planet formation. One of the fascinating areas to explore in the coming decade is how the final composition of a planet may be influenced by its formation environment. The origin of water on our own planet is a key example, but similar questions exist regarding the disposition of water on Jupiter.

Edwin A. Bergin

University of Michigan, Department of Astronomy, 1085 S. University Ave., Ann Arbor, MI, 48109, USA. e-mail: ebergindumich.edu

L. Ilsedore Cleeves

Harvard-Smithsonian Center for Astrophysics, 60 Garden Street, Cambridge, MA 02138. e-mail: ilse.cleeves@cfa.harvard.edu 
To make these links, high signal-to-noise spectra of exoplanetary atmospheres and the continuing study of the solar system record at all scales are necessary. However, we also need to have fundamental knowledge of how chemistry evolves before, during, and after planetary birth. The focus of this chapter is to provide a baseline for the key chemical processes during the gas-rich stages of planet formation, including recent updates within this fast-moving field.

There have been a number of reviews of disk chemistry over the past decade (Bergin et al. 2007; Henning and Semenov 2013, Dutrey et al.|2014), and the goal of this chapter is not to provide a complete review of the field. Rather, we aim our discussion towards new researchers in the relevant fields with references that link back to the grounding datasets, laboratory data, and/or theoretical underpinnings. An important aspect of this volume is the discussion of new advances brought about by ALMA, which are still unfolding. Fig. 1 presents a montage of molecular observations made with ALMA towards various disks of different masses, ages, and host stars. One new aspect is the ubiquity of radial structure in the images with differences between species even when observed in the same source (e.g. $\mathrm{C}_{2} \mathrm{H}, \mathrm{C}^{18} \mathrm{O}$, $\mathrm{CN}$, and $\mathrm{N}_{2} \mathrm{H}^{+}$are each observed towards TW Hya). This hints at an evolving and rich active chemistry which is responding to physical changes induced in the gas or the dust.

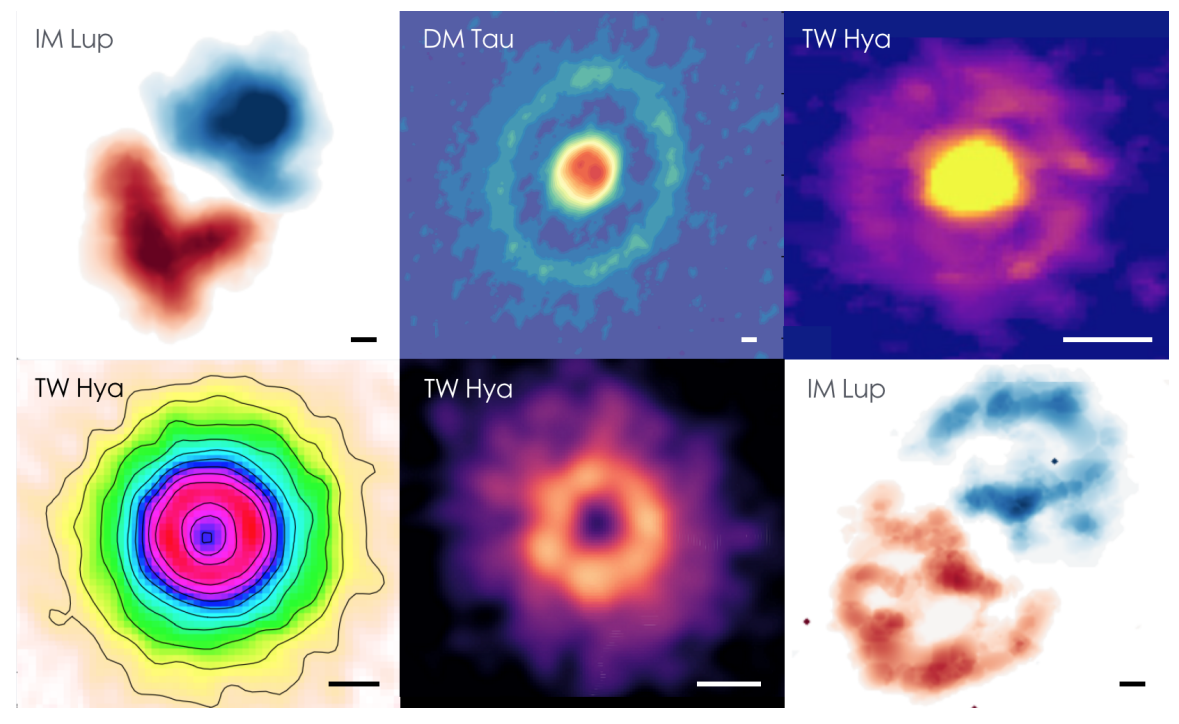

Fig. 1 Molecular observations in disks exhibiting a wide variety of ring-like structure. Sources labeled in the top left and a $50 \mathrm{AU}$ scale bar shown in the bottom right of each panel. From left to right and top to bottom: $\mathrm{H}^{13} \mathrm{CO}^{+}$(Öberg et al. 2015), $\mathrm{C}_{2} \mathrm{H}$ (Bergin et al. 2016), ${ }^{13} \mathrm{CO}$ (Schwarz et al. 2016), $\mathrm{CN}$ (Teague et al. 2016), $\mathrm{N}_{2} \mathrm{H}^{+}$(data from Qi et al.2013), $\mathrm{DCO}^{+}$(Oberg et al.2015), reproduced with permissions.

The chapter begins by detailing the evolving physical environment (dust/gas density and temperature, radiation field). We then explore disk chemistry within this environment in three sections, in each case providing separate theoretical and ob- 
servational perspectives. These sections begin with the dense, shielded midplane with a strong focus on snow lines. We then discuss the "warm molecular layer," where the conditions are warm enough such that molecules exist in gaseous form above a frozen midplane (Aikawa et al. 2002). Finally, we explore the atomic-tomolecular transition at the disk surface itself. We conclude by discussing how the disk composition might relate to overall planetary composition for both rocky and Jovian-like worlds.

\section{The Physical Environment}

Decades of protoplanetary disk study have isolated several salient facts regarding the disk physical properties that have strong influence on the resulting chemical composition. In Fig. 2 we show a generalized schematic of the relevant physical processes and their location(s) of influence.

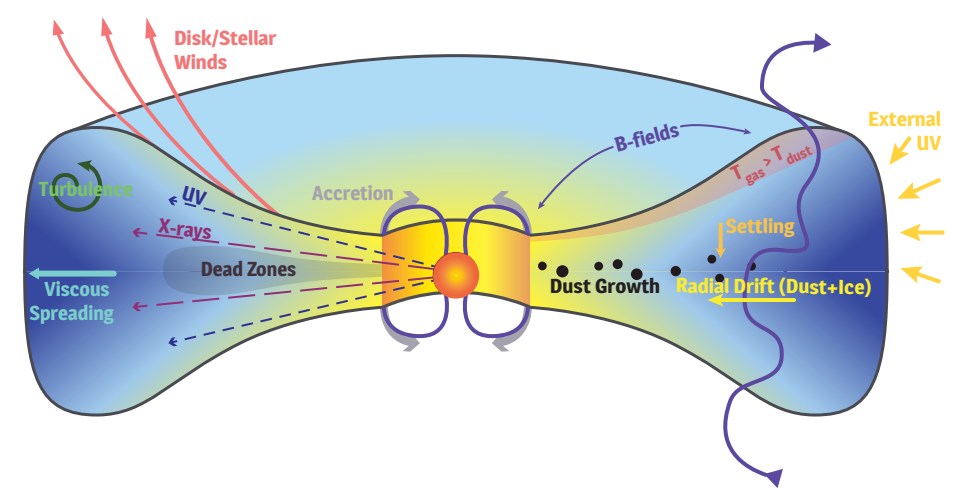

Fig. 2 Illustration of key physical processes in protoplanetary disks. Reproduced with permission from Cleeves 2015 (PhD Thesis).

Gas Density Distribution: early estimates of the gas density structure relied on a posterior distribution of planetary mass, adjusted by solar elemental abundances relative to hydrogen. This calculation naturally leads to a radial fall-off of the overall gas surface density profile as $\Sigma(R) \propto R^{-1.5}$, a distribution referred to as the "minimum mass solar nebula" (Hayashi 1981, Weidenschilling 1977). To achieve consistency with the overall dust spectral energy distribution, modern models of disk evolution balance the effects of the irradiation of the central star dominated by the radiation at the blackbody peak, and that of hydrostatic equilibrium of the heated dust surface (Kenyon and Hartmann 1995, Calvet et al. 1991). A critical factor in this calculation is the disk viscosity, which is generally captured by the traditional $\alpha$ parameter which relates the turbulent viscosity $(v)$ to disk motions, $v=\alpha c_{s} / \Omega_{K}$ (Shakura and Syunyaev 1973). Here $c_{s}$ is the isothermal sound speed and $\Omega_{K}$ the Keplerian rotation speed which will vary throughout the disk. Fig 3 presents an ex- 
ample of a typical observationally motivated gas density structure. More detailed discussion regarding gas disk evolution and observational constraints can be found in the recent review of Bergin and Williams (2017).
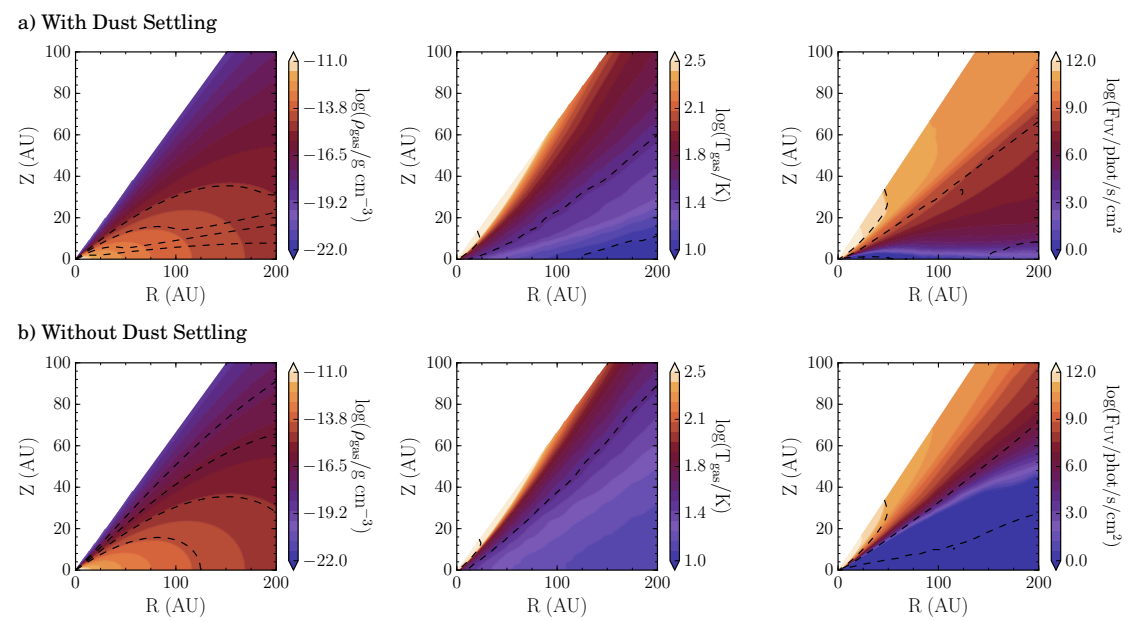

Fig. 3 Example disk model for a gas-rich disk around a $1 \mathrm{M}_{\odot}$ star a) with settling (top) and b) without settling (bottom). Left panels are gas density, over-plotted with dust density contours as labeled. Middle panel is gas temperature overlaid with dust temperature contours. Right show the UV flux, overlaid with contours of the X-ray flux.

Dust Density Distribution: Observations of resolved sub-millimeter thermal dust emission continuum maps (e.g. Isella et al. 2009; Andrews et al.|2011), the overall dust spectral energy distribution (e.g. Furlan et al. 2005), and scattered light images (e.g. Debes et al. 2013) have now established that the dust has at least two major distributions. Small (sub)-micron sized grains, responsible for much of the spectral emissions in the near and mid-infrared and for the absorption of high energy radiation, are dynamically coupled to the gas and have a spatial distribution that is comparable to that of carbon monoxide emission. As grains grow they are subject to differential forces that lead to both settling and radial drift towards gas pressure maxima (i.e. the midplane and the inner disk; Whipple 1973, Weidenschilling and Cuzzi 1993, Dullemond and Dominik 2004). In the latter case, drift is prevalent for sizes that are large enough to be decoupled from the gas $(\geq 1 \mathrm{~mm})$ but small enough that they feel effects of gas pressure $(<$ few $\mathrm{km})$ depending on disk properties and orbital distance (Weidenschilling and Cuzzi 1993). Correspondingly, larger grains, which carry the disk dust mass have a distribution that is spatially concentrated. In the radial direction the large grains will drift into the inner tens of AU disk or perhaps greater distances, but are smaller than the $\mathrm{CO} /$ small grain scattered light disk. In the vertical direction models suggest the grains exist in a very thin $(\sim 1 \mathrm{AU})$ layer (Pinte et al.2016). These issues are directly addressed in the chapter by Andrews \& Birnstiel. 
High-Energy Radiation Field: The main chemical pathways in dense gas at low temperatures $(\sim 10-100 \mathrm{~K})$ are through exothermic reactions between ions and neutrals (Herbst and Klemperer 1973). Thus, the gas-phase chemistry is powered by ionizing sources. For disks there are several important contributors to the overall ionization structure: UV and X-ray radiation (both internal and external) and galactic cosmic rays.

1. Galactic cosmic rays, with an ionization rate in dense gas of $\zeta_{\mathrm{H} 2} \sim 5 \times 10^{-17} \mathrm{~s}^{-1}$ (Dalgarno 2006), have the greatest penetration power, reaching gas at column densities up to $\Sigma_{C R}=96 \mathrm{~g} \mathrm{~cm}^{-2}$ as given by Umebayashi and Nakano (1981) (but see updates in Padovani et al. 2009, Cleeves et al. 2013). Recent work by Cleeves et al. (2013) shows that the lower energy cosmic rays responsible for ionization might be impeded by stellar winds in a similar manner as in our own solar system (Webber 1998). Winds can reduce the net low energy particle flux by many orders of magnitude, reducing the cosmic ray ionization rate by one or more orders of magnitude (Cleeves et al. 2013).

2. X-rays are an important source of deep ionization, while providing a steady productive source of ionization for the disk surface. Young low-mass stars, such as our Sun, are uniformly associated with stellar magnetic activity and active solarlike X-ray generating corona, and perhaps accretion. The X-ray luminosity can vary with median values of $\sim L_{X} \sim 10^{30} \mathrm{~s}^{-1}$ (Güdel 2004) with typical X-ray temperatures of $k T_{X} \sim 1 \mathrm{keV}$ (Preibisch et al. 2005). Note there is also harder $\mathrm{X}$-ray component with $k T_{X}>3 \mathrm{keV}$ that may be associated with flare activity (Preibisch et al. 2005). X-ray photon propagation is governed by photoabsorption due to heavy elements (Morrison and McCammon 1983: Henke et al. 1993), with scattering of increasing import above $5 \mathrm{keV}$ (Igea and Glassgold 1999). For reference, normalized to solar abundances, the cross-section per hydrogen at 1 $\mathrm{keV}$ is $\sigma_{1 \mathrm{keV}}=2 \times 10^{-22} \mathrm{~cm}^{-2}$ while at $5 \mathrm{keV}, \sigma_{5 \mathrm{keV}}=2 \times 10^{-24} \mathrm{~cm}^{-2}$ (Morrison and McCammon 1983, Bethell and Bergin 2011a). As a consequence, there is a significant increase in penetration depth with energy (Igea and Glassgold 1999). Dust growth, settling, and drift can influence the X-ray radiation transfer with a minimum value set by volatile gas-phase absorbers (e.g. H, C, O; Bethell and Bergin 2011a).

3. Ultraviolet Radiation (UV) is dominated by accretion luminosity for low-mass cool stars (color temperatures $<6000 \mathrm{~K}$ ) and by the stellar photosphere for $\mathrm{F}$ stars and above. The typical FUV luminosity for accreting (Classical T Tauri stars) young stars is $10^{-3}-10^{-1} \mathrm{~L}_{\odot}$; stars with little accretion, so called weak line T Tauri stars, have values of $L_{F U V} \sim 10^{-6}-10^{-4} L_{\odot}$ (Yang et al. 2012). For low mass stars the FUV luminosity has several components: an underlying continuum attributed to the accretion shock (Gullbring et al. 2000), strong - but absorbed - photospheric Ly $\alpha$ emission (Herczeg et al. 2002; Schindhelm et al. 2012), lines from highly ionized atoms (Ardia et al. 2013), and a forest of $\mathrm{H}_{2}$ emission lines (France et al. 2012). Ultraviolet radiation has the smallest penetration depth $\sigma_{F U V} \sim 10^{-3} \mathrm{~g} \mathrm{~cm}^{-2}$, but it has a strong dependence on grain evolution as its propagation is governed by smaller $0.1 \mu \mathrm{m}$ grains that grow and settle to the midplane. Thus, models of UV radiation transfer show that it is 
highly dependent on the photoabsorption and scattering properties of evolving dust grains (van Zadelhoff et al. 2001). Of particular importance is the strong Ly $\alpha$ radiation which dominates the FUV field containing 70-90\% of the FUV luminosity (Herczeg et al. 2002, Schindhelm et al. 2012). Detailed models show that, due to $\mathrm{H}$-atom scattering in the upper layers, Ly $\alpha$ photons have greater penetrating power than the other components of the FUV spectrum (Bethell and Bergin 2011b); this has an imprint on the resulting chemistry (Fogel et al. 2011).

Temperature: Stellar radiation and heating from accretion dominate the thermal budget of disk systems. Calvet et al. (1991) demonstrated that this heating leads to a temperature inversion on the disk surface where the disk midplane is colder then the surface with the superposition of an overall radial temperature gradient (e.g. Fig. 2 and Fig. 3). This temperature profile has direct implications for the overall spectral emissions from dust (Chiang and Goldreich 1997, D’Alessio et al. 1999, 2001) and also gas emission (van Zadelhoff et al.|2001; Aikawa et al.|2002). From the chemical perspective, the most general consequence is that there will be at least two sublimation fronts, e.g., snowlines, see the Midplane and Molecular Layer sections below. In addition, the evolution of the dust population (settling and drift) will also strongly effect the thermal structure, where a more settled disk tends to be globally warmer (Fig. 3 and see also D'Alessio et al. 2005, Cleeves 2016; Facchini et al. 2017).

\section{Disk Chemistry by Environment}

Below we divide the disk into 3 vertical zones that delineate key chemical transitions in the disk. These are as follows. The disk midplane is defined as the layer with the maximum of the gas density distribution in the vertical direction. With high densities, fast gas-dust collision timescales, and decaying temperatures with distance from the star, this layer is dominated by sublimation fronts and gas/ice transitions in the radial direction. The next vertical zone, the so-called "warm molecular layer" (Aikawa et al.2002), is defined by a chemical definition as the layer where the vertical dust temperature exceeds that required for sublimation of $\mathrm{CO}$, the most volatile carrier of an abundant heavy element $(\sim 20 \mathrm{~K})$. The top of this layer is defined by the zone where molecules will be photo-dissociated at $\tau_{u v} \sim 1$. This layer has an active chemistry with ionizing agents, such as X-rays (and/or cosmic rays), facilitating ionmolecule chemistry in UV-shielded gas. The last vertical zone refers to the stellarirradiation dominated surface and the "atomic-to-molecular transition". This layer is defined by having $\tau_{u v} \leq 1$ whereupon there is an interplay between molecular dissociation and reformation of predominantly simple molecules with fast formation rates. 


\section{Midplane}

\section{Theory}

The plane that intersects the geometrical center of the disk perpendicular to the rotation axis is referred to as the disk "midplane". The midplane is enhanced in dust that has grown and settled from the surface. In the midplane, the dust can further dynamically evolve and grow and eventually form planetesimals (see Andrews \& Birnstiel chapter). Thus the composition and conditions of the midplane are of particular interest for understanding the initial formation properties of planetesimals.

The midplane is relatively cooler and more shielded from radiation compared to the disk surface at a given radius. The temperature in the midplane is regulated by gas accretion close to the star (within one to a few $\mathrm{AU}$ ), and from reprocessed stellar radiation intercepted by small dust grains in the upper layers of the disk as discussed above. These small dust grains indirectly heat the disk midplane beyond a few AU. The densities are typically sufficiently high such that the gas temperature is equal to that of the dust due to frequent collisions between the two populations.

Of particular importance to the midplane composition are the strong chemical transitions from the ice to vapor, or snowlines. Such locations have been posited as important sites for dust grown or planet formation (e.g., Stevenson and Lunine 1988). At sufficiently cold temperatures, the gas begins to "freeze-out" onto dust surfaces once the rate of molecules hitting the surface matches the rate of molecules thermally desorbing (sublimating) from the surface. Following the formalism of Hollenbach et al. (2009), the thermal desorption rate $\left(R_{t d}\right)$ per atom or molecule is given by,

$$
R_{t d, i} \simeq v_{i} e^{-E_{a, i} / k T_{g r}} .
$$

Here, $v_{i}=1.6 \times 10^{11} \sqrt{\left(E_{a, i} / k\right) /\left(m_{i} / m_{\mathrm{H}}\right)} \mathrm{s}^{-1}$ is the vibrational frequency of the species in the surface potential well with $m_{i}$ and $m_{h}$ the mass of species $i$ and hydrogen; $k$ is the Boltzmann constant. $E_{a, i}$ is the binding energy of species $i$ to the surface. To determine the sublimation temperature of a molecule from a given surface we balance the desorption rate with the flux of molecules that are absorbing from the gas $\left(F_{a b}\right)$.

Thus,

$$
F_{a b, i} \equiv N_{s} R_{t d, i} f_{s, i}=0.25 n_{i} v_{i} S,
$$

$\mathrm{N}_{s}$ equal to number of surface sites available per $\mathrm{cm}^{2}\left(N_{s} \sim 10^{15}\right.$ sites $\mathrm{cm}^{-2}$, for a $0.1 \mu \mathrm{m}$ grain) and $\mathrm{f}_{s, i}$ the fraction of those sites occupied by species $i . n_{i}$ is the space density of species $i, v_{i}$ its thermal velocity, and $\mathrm{S}$ is the sticking coefficient which is generally assumed to be unity. Solving for the grain temperature we will determine the sublimation temperature, $T_{s u b}$ for a given species: 
$T_{s u b, i} \simeq \frac{E_{a, i}}{k}\left[57+\ln \left[\left(\frac{N_{s, i}}{10^{15} \mathrm{~cm}^{-2}}\right)\left(\frac{v_{i}}{10^{13} \mathrm{~s}^{-1}}\right)\left(\frac{1 \mathrm{~cm}^{-3}}{n_{i}}\right)\left(\frac{10^{4} \mathrm{~cm} \mathrm{~s}^{-1}}{v_{i}}\right)\right]\right]^{-1}$.

Because desorption is balanced by the absorbing rate the sublimation temperature (for a given binding energy) will have a dependence on the local gas pressure. In addition, a particular molecule's adsorption energy can additionally depend on the properties of the grain surface. For example, highly polar water increases the binding energy of $\mathrm{CO}$ to water ice to a value of $E_{a} \sim 1500 \mathrm{~K}$ (Fayolle et al.2016), compared to $\mathrm{CO}$ bound to pure $\mathrm{CO}$ ice, which has $E_{a}=855 \mathrm{~K}$ (Öberg et al. 2009). For a pressure of $10^{-10} \mathrm{bar}$, this corresponds to a difference in absolute desorption temperature of $19 \mathrm{~K}$ and $26 \mathrm{~K}$, respectively.

Figure 4 illustrates the interdependence of gas pressure and dust temperature in setting location of various molecules' snowlines, along with a "typical" midplane temperature profile for a disk around a solar-mass star. Molecules such as $\mathrm{H}_{2} \mathrm{O}$, $\mathrm{CH}_{3} \mathrm{OH}$, and $\mathrm{NH}_{3}$ have the highest $E_{a}$, and thus tend to remain in the ice phase until the temperatures are very warm. Molecules such as $\mathrm{N}_{2}$ and $\mathrm{CO}$ have smaller $E_{a}$, and correspondingly they remain in the gas phase for tens of $\mathrm{AU}$ in radius for a solar mass star.

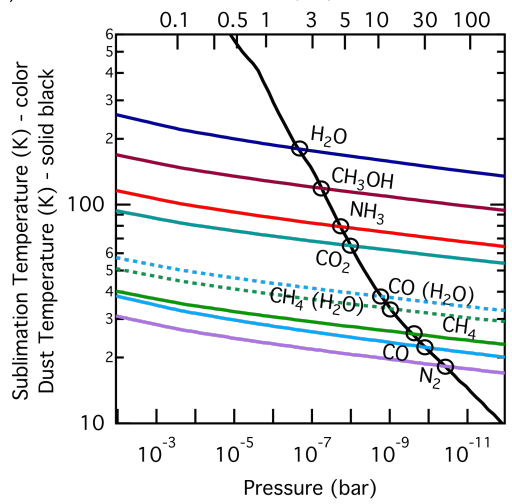

b)

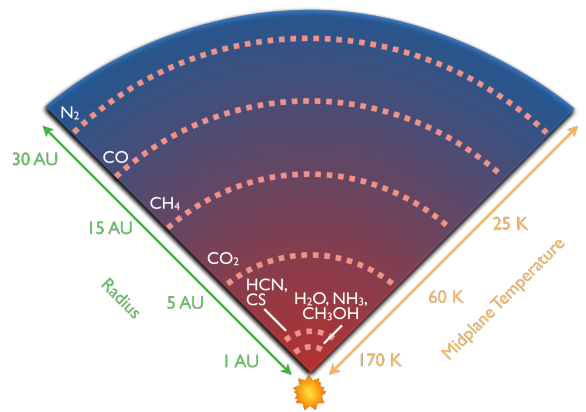

Fig. 4 (Left:) Sublimation temperature of various molecules onto surfaces dominated by pure ices (i.e. same species) or water as a function of gas pressure. Binding energies are from laboratory work with the following references, $\mathrm{N}_{2}$ : Fayolle et al. (2016), $\mathrm{CO}$ : Fayolle et al. (2016), $\mathrm{CH}_{4}$ : Herrero et al. (2010), $\mathrm{CH}_{4}-\mathrm{H}_{2} \mathrm{O}$ : Behmard et al. 2018 (in prep.), $\mathrm{CO}-\mathrm{H}_{2} \mathrm{O}$ : Cleeves et al. (2014), $\mathrm{CO}_{2}$ : Martín-Doménech et al.(2014), $\mathrm{NH}_{3}$ : Martín-Doménech et al.(2014), $\mathrm{CH}_{3} \mathrm{OH}$ : Martín-Doménech et al. (2014), $\mathrm{H}_{2} \mathrm{O}$ : Fraser et al. (2001). The solid line is one realization of a pressure-temperature profile in the midplane taken from D'Alessio et al. (2006). (Right:) Illustration of midplane freezeout of various molecules with distance from the star (decreasing temperature). 


\section{Observations}

Most of the midplane gas is observationally "hidden." The temperatures are too low in the outer disk (beyond tens of AU) for most observable species to remain in the gas, while those that do are often so cold the observable transitions are not strongly excited, or are obscured by warmer gas in the surface. In the inner disk, the high column densities present in disks make many observable transitions optically thick, also acting to "hide" midplane gas from our view. Furthermore, dust opacity from settled millimeter grains can act to obscure molecular emission from the midplane itself. One exception is that of ${ }^{13} \mathrm{C}^{18} \mathrm{O}$ whose low column density $(\sim 30,000$ times less than ${ }^{12} \mathrm{CO}$, allows it to be observed down to the midplane inside of its snow line (Zhang et al.2017), see also Figure 5 .

There have been many major observational efforts to isolate the solid-to-gas phase transitions (snowlines) in the midplane. At present, the most observationally accessible snowline is that of $\mathrm{CO}$. This feature is a direct consequence of CO's low $E_{a}$, and thus the $\mathrm{CO}$ snowline occupies tens of AU scales that are more readily observationally accessible than that of, e.g., $\mathrm{H}_{2} \mathrm{O}$ (which will lie within $\sim 1 \mathrm{AU}$ for a solar type star. The primary tracers of the $\mathrm{CO}$ snowline that have been used in the literature include $\mathrm{N}_{2} \mathrm{H}^{+}, \mathrm{DCO}^{+}$, and optically thin isotopologues of $\mathrm{CO}$.
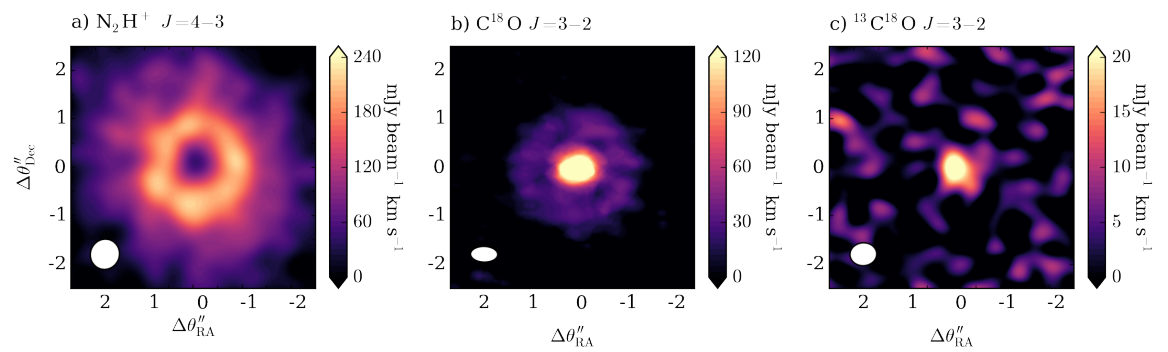

Fig. 5 Observational tracers of the CO snow line. Data from Qi et al. (2013), Schwarz et al. (2016), and Zhang et al. (2017), left to right.

$\mathrm{N}_{2} \mathrm{H}^{+}$: The chemistry of $\mathrm{N}_{2} \mathrm{H}^{+}$is such that it is rapidly destroyed in the presence of CO. As a consequence, $\mathrm{N}_{2} \mathrm{H}^{+}$has been historically used as a marker of $\mathrm{CO}$ freeze-out in the dense interstellar medium (Charnley 1997; Bergin et al. 2002). $\mathrm{N}_{2} \mathrm{H}^{+}$shows a clear ring-like distribution in the TW Hya protoplanetary disk (see Figure 5) with an inner radius of $\sim 30$ AU. Correspondingly, the Qi et al. (2013) paper interpreted the ring of $\mathrm{N}_{2} \mathrm{H}^{+}$as a marker of the TW Hya disk's $\mathrm{CO}$ snow line, which was also in reasonable agreement with model temperature estimates of the disk at these radii (Qi et al. 2013, 2015).

CO Isotopologues: More recently, measurements with ALMA of less abundant (and less optically thick) $\mathrm{CO}$ isotopologues found a steep drop off of $\mathrm{C}^{18} \mathrm{O}$ intensity at $\sim 17-23 \mathrm{AU}$, interior to the $\mathrm{N}_{2} \mathrm{H}^{+}$ring (Schwarz et al. 2016). These results were more recently supported by ${ }^{13} \mathrm{C}^{18} \mathrm{O}$ observations, which place the column density break at a radius of $20.5 \pm 1.3 \mathrm{AU}$ (Zhang et al. 2017). The break is attributed to freeze out at the midplane $\mathrm{CO}$ snow line. 
This $\sim 10 \mathrm{AU}$ spatial discrepancy between the $\mathrm{N}_{2} \mathrm{H}^{+}$transition and the $\mathrm{CO}$ transition is in part due to the inescapable nature of the disk temperature gradients, which are not purely radial. Instead, the vertical increase in temperature with height in the disk (see Figure 6) causes the region of $\mathrm{CO}$ freeze-out to occupy a wedge, with $\mathrm{N}_{2} \mathrm{H}^{+}$in the simplest case bounding its borders until $\mathrm{N}_{2}$ freeze-out commences. More detailed modeling of the chemistry (e.g., Aikawa et al. 2015, van't Hoff et al. 2017) shows that the distribution of $\mathrm{N}_{2} \mathrm{H}^{+}$is complicated by additional factors, including the desorption rates of $\mathrm{CO}$ vs. $\mathrm{N}_{2}, \mathrm{CO}$ abundance, and disk ionization.

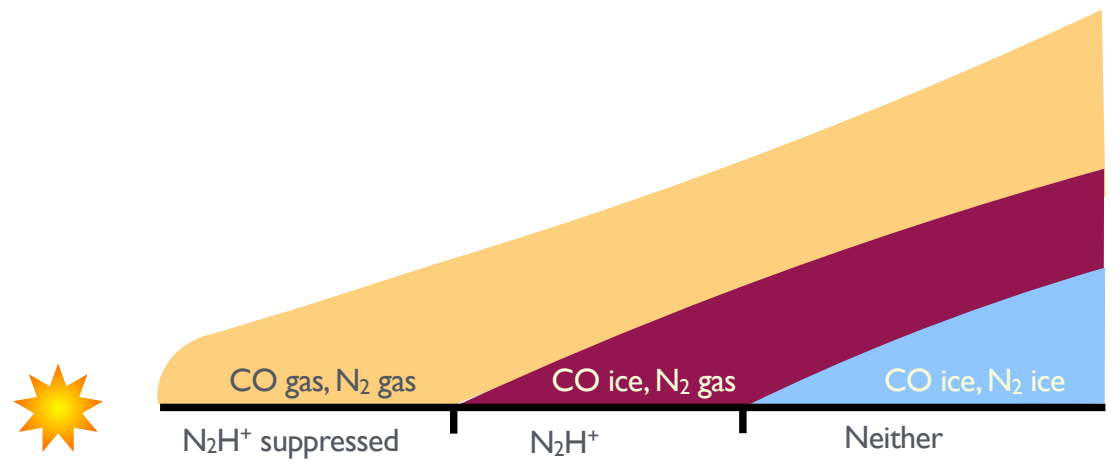

Fig. 6 Schematic of the "classical" picture of the relationship between $\mathrm{N}_{2} \mathrm{H}^{+}$and the $\mathrm{CO}$ snow line. $\mathrm{N}_{2}$ is expected to stay in the gas down to lower temperatures than $\mathrm{CO}$, leading to a layer between the region of $\mathrm{CO}$ freeze out and $\mathrm{N}_{2}$ freeze out where $\mathrm{N}_{2} \mathrm{H}^{+}$is expected to occupy.

\section{Molecular Layer}

\section{Theory}

In between the extremes of freeze-out and dissociation by ultraviolet photons, there exists a layer rich in gas-phase molecules, the "warm molecular layer" Aikawa et al. 2002). This layer does not have discrete boundaries, but rather contains a mixture of neutral and ionized molecules and more strongly bound ices like water and ammonia. It is shielded sufficiently from strong UV irradiation to not fully destroy newly formed molecules, yet enough UV penetrates to facilitate a rich radical-driven chemistry. Within this layer, the disk transitions from being optically thin to X-rays to becoming thick, and can sustain a rich ion-neutral gas phase chemistry. Simultaneously, the temperatures are becoming cool enough such that molecules adhere to the surface of the cold dust grains $\left(T_{\text {dust }}<50 \mathrm{~K}\right)$ long enough to initiate grain surface chemistry (e.g., Loomis et al.|2015, for $\mathrm{H}_{2} \mathrm{CO}$ ). In terms of building toward molecular complexity, these factors make the warm molecular layer a highly chemically active region in disks. 

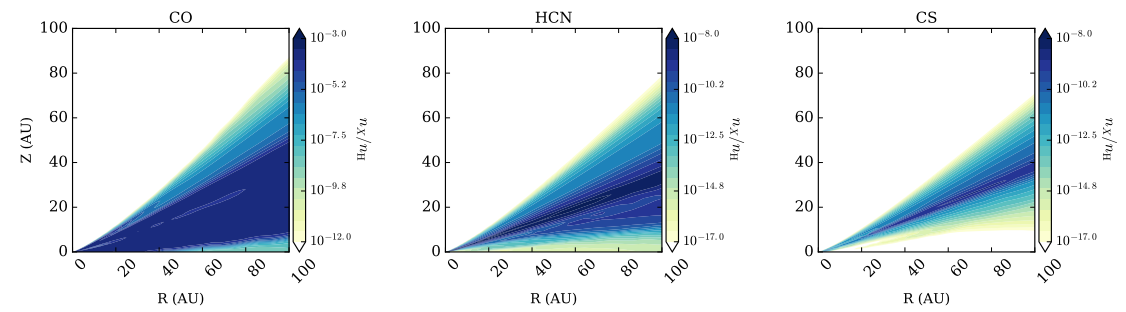

Fig. 7 Example molecular distributions of key molecules known to be abundant in disks, CO, HCN, and CS. Model physical structure from Figure 3 Taken from Cleeves (2016).

Figure 7 shows typical chemical model abundances for three commonly observed molecules in disks, $\mathrm{CO}, \mathrm{HCN}$, and CS, for a disk around a solar mass star (taken from Cleeves 2016). CO is the second most abundant small molecule in the interstellar medium (after $\mathrm{H}_{2}$ ), which is in part a testament to its robust chemistry. As is shown in the Figure, $\mathrm{CO}$ is present in the gas phase for a large radial and vertical portion of the disk. Over time, $\mathrm{CO}$ tends to form molecules like $\mathrm{CH}_{3} \mathrm{OH}$ and $\mathrm{CO}_{2}$, which have higher binding energies. These molecules freeze-out onto small dust grains removing some amount of $\mathrm{CO}$ (see, e.g. Bergin et al. 2014, Furuya and Aikawa 2014, Reboussin et al. 2015). This behavior produces some of the banded structure in Figure 7

All three molecules have a steep abundance drop off in the upper atmosphere, traced by $z / r \gtrsim 0.5$, corresponding roughly to the $\tau_{\mathrm{UV}} \sim 1$ surface. At the lower bound, the molecules begin to freeze-out. In these particular models, the assumed binding energy of CS is greater than $\mathrm{HCN}$, causing CS to freeze out at higher altitudes than HCN, placing it in a more narrow layer. These illustrative cases demonstrate that the vertical distribution of molecules is very sensitive to the temperature and density gradients, through freeze out and high energy radiation opacity.

The molecular layer is perhaps most strongly subject to (and in some ways, responsible for) the time-evolving chemical effects of dust growth and settling. Icecoating, especially that of water, aids in the probability of grains sticking together upon collision (e.g. Wang et al. 2005). As grains grow and decouple from the gas, the process of settling naturally displaces volatiles. In the absence of vigorous mixing, the process preferentially removes volatiles from the surface, sequestering the ices into the midplane, where they can further dynamically evolve. As water is a dominant ice constituent both in the interstellar medium, comets, and likely disks (van Dishoeck et al. 2013a), this process will tend to deplete oxygen to a greater extent than carbon, raising the carbon to oxygen ratio of the gas in the surface. As a result, the gas-phase chemistry will tend toward forming abundant carbon rich molecules (e.g., hydrocarbons and cyanides, see Figure 8). 


\section{Observations}

As this region is rich in gas-phase molecules, most of our observations of the molecular content of disks come from the warm molecular layer. For edge-on disks, the vertical stratification can be directly observed (see Figure 9). In addition to $\mathrm{CO}$, a host of additional molecules have been observed in disks. In the submillimeter, detected molecules include: $\mathrm{CO},{ }^{13} \mathrm{CO}, \mathrm{C}^{18} \mathrm{O},{ }^{13} \mathrm{C}^{18} \mathrm{O}, \mathrm{HCO}^{+}, \mathrm{DCO}^{+}, \mathrm{H}^{13} \mathrm{CO}^{+}$, $\mathrm{HC}^{18} \mathrm{O}^{+}, \mathrm{CN},{ }^{13} \mathrm{CN}, \mathrm{C}^{15} \mathrm{~N}, \mathrm{HCN}, \mathrm{DCN}, \mathrm{H}^{13} \mathrm{CN}, \mathrm{HC}^{15} \mathrm{~N}, \mathrm{CS}, \mathrm{SO}, \mathrm{N}_{2} \mathrm{H}^{+}, \mathrm{N}_{2} \mathrm{D}^{+}$, $\mathrm{C}_{2} \mathrm{H}, \mathrm{C}_{2} \mathrm{~S}, \mathrm{H}_{2} \mathrm{CO}, \mathrm{HC}_{3} \mathrm{~N}, c-\mathrm{C}_{3} \mathrm{H}_{2}, \mathrm{CH}_{3} \mathrm{CN}, \mathrm{CH}_{3} \mathrm{OH}$. In the far-infrared, $\mathrm{HD}, \mathrm{H}_{2} \mathrm{O}$, $\mathrm{OH}, \mathrm{NH}_{3}, \mathrm{CH}^{+}$and mid/near infrared, $\mathrm{H}_{2}, \mathrm{C}_{2} \mathrm{H}_{2}, \mathrm{H}_{2} \mathrm{O}, \mathrm{HCN}, \mathrm{CO}_{2}$, and $\mathrm{CH}_{4}$. In addition, many of these molecules are not homogeneously distributed through the disk as demonstrated in Figure 1.

Consistent with the picture of time-depletion of oxygen relative to carbon, hydrocarbon rings have been demonstrated to be particularly bright (Kastner et al. 2015, Bergin et al. 2016). Thus, while the surface layers are undergoing active chemistry, along with volatile depletion, by studying the chemistry of the surface one can begin to learn indirectly about what is locked up, hidden from observations, in the midplane.

\section{Atomic-to-Molecular Transition}

\section{Theory}

The disk surface is highly exposed to energetic radiation from the star which is dominated by both the stellar far-UV (FUV: $912 \AA<\lambda<2000 \AA$ ) radiation and X-

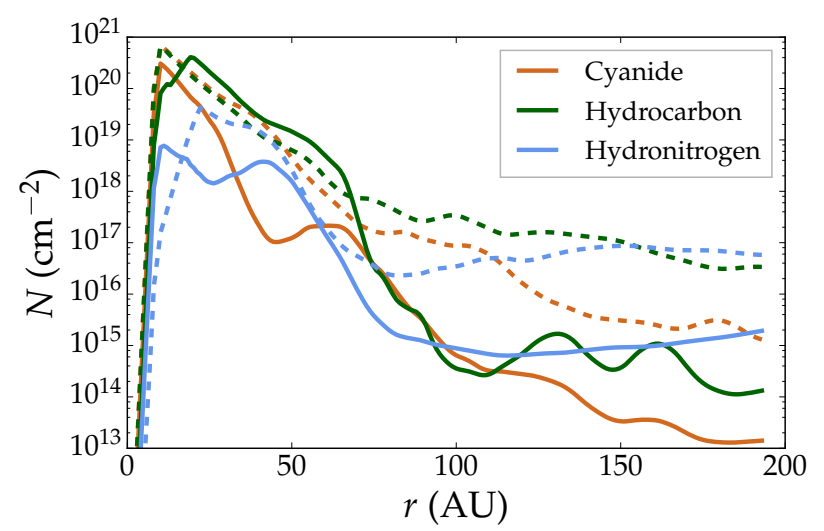

Fig. 8 Simulated column densities with radial distance from the star for the molecular families (molecules with $\mathrm{C}-\mathrm{N}$ bonds, $\mathrm{C}-\mathrm{H}$ bonds, and $\mathrm{N}-\mathrm{H}$ bonds) as indicated in the legend. The solid lines show models with interstellar carbon and oxygen abundances, while the dashed lines show models that have reduced carbon and oxygen abundances. Reproduced with permission from $\mathrm{Du}$ et al. (2015) 
rays. This surface draws parallels with regions in the dense $\left(>10^{5} \mathrm{~cm}^{-3}\right)$ interstellar medium that are irradiated by nearby massive stars, i.e., photodissociation regions or PDRs (Hollenbach and Tielens 1999). Indeed, early work on chemical modeling of PDRs provide the fundamental physical basis (e.g., through the micro-physics of heating and cooling) for thermal-chemical models of disks (Kamp and Dullemond 2004, Gorti and Hollenbach 2004, Nomura and Millar 2005; Woitke et al. 2009, Du and Bergin 2014: Ádámkovics et al. 2014).

In interstellar PDRs the strength of the radiation field is generally given in units of the local interstellar radiation field. This is determined by computing the radiation generated by massive stars in the solar vicinity; this has a value estimated to be $G_{0}=1.6 \times 10^{-3} \mathrm{erg} \mathrm{cm}^{-2} \mathrm{~s}^{-1}$ (Habing 1968). A typical UV radiation field of an accreting T Tauri star has a value of $G \sim 500 G_{0}$ at $100 \mathrm{AU}$ (Bergin et al. 2004; Yang et al. 2012). The dense $\left(\mathrm{n}>10^{6} \mathrm{~cm}^{-3}\right)$ disk close to the star thus has warm, $\mathrm{T}_{\text {gas }} \sim 400-1000 \mathrm{~K}$, material in surface layer and a very hot disk photosphere $(>$ $1000 \mathrm{~K})$ with cool $(\leq 20 \mathrm{~K})$ very dense material over a hundred AU from the star. This temperature and opacity profile produces important differences in the relative chemical transitions, compared to ISM PDRs, which are illustrated in Fig. 10 and summarized below.

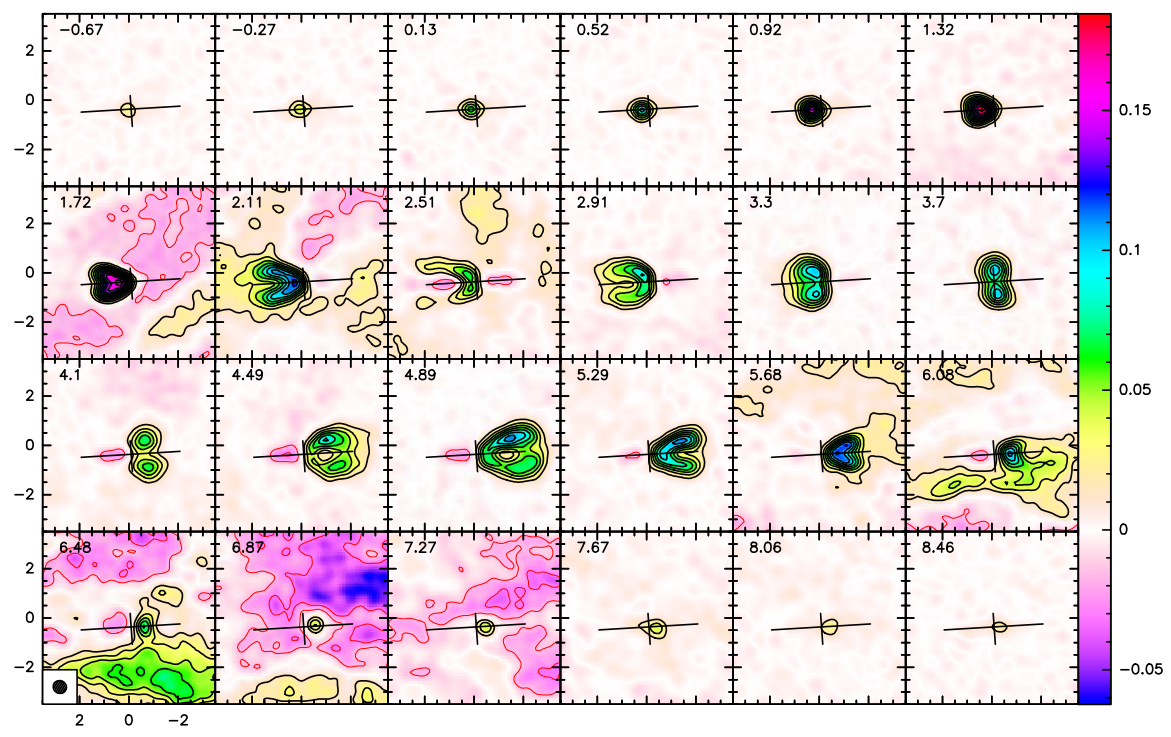

Fig. 9 A protoplanetary disk viewed edge on in ${ }^{12} \mathrm{CO} J=2-1$. Reproduced with permission from Dutrey et al. (2017). Each of these panels represents the emission seen in a velocity channel corresponding to the number in $\mathrm{km} \mathrm{s}^{-1}$ on the top right. The systemic velocity is near $3.7 \mathrm{~km}$ $\mathrm{s}^{-1}$. Clear evidence is seen for rotation as the highest velocities correspond to the inner disk. The horizontal line shows the midplane angle and note the absence of emission from distant disk layers $\left(>1^{\prime \prime}\right)$ in the midplane at velocities near $2-3 \mathrm{~km} \mathrm{~s}^{-1}$ and $4-5 \mathrm{~km} \mathrm{~s}^{-1}$. This missing emission is visual evidence for the $\mathrm{CO}$-ice dominated zone. 


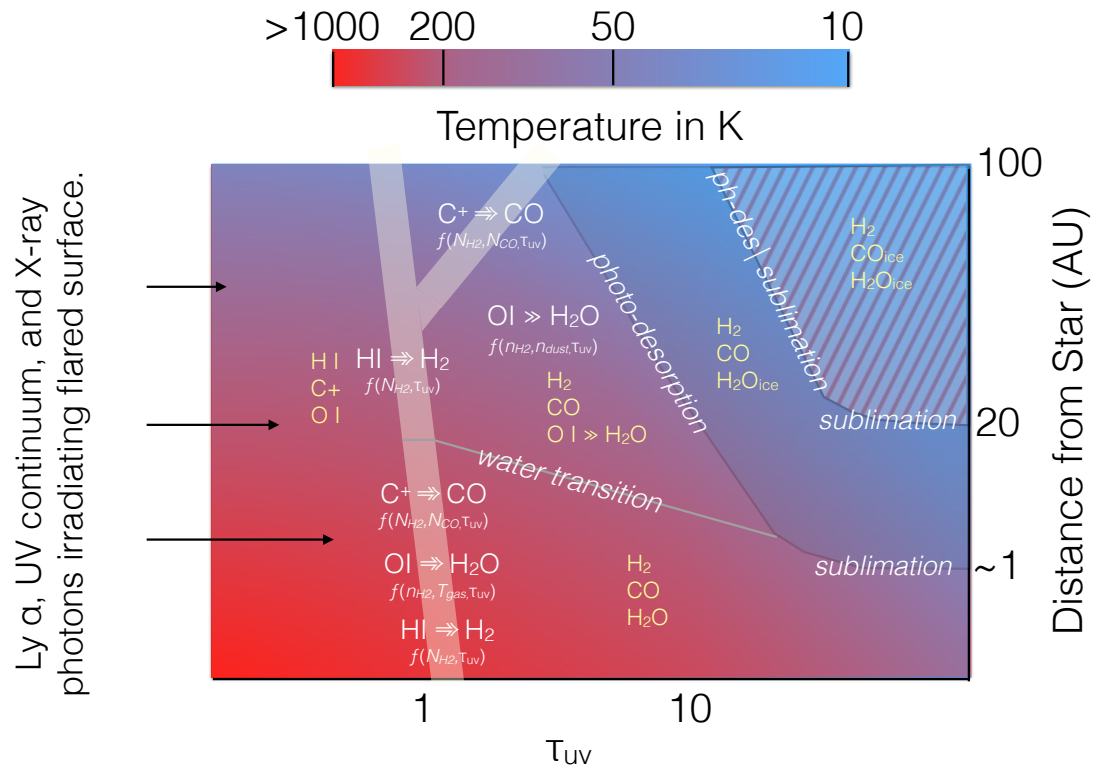

Fig. 10 Schematic of the disk PDR surface illustrating key atomic-molecular transitions. The width of the gray bars show the rough location of each transition. $\mathrm{H}_{2}$ and $\mathrm{CO}$ have transitions that are mediated by self-shielding. The water transition has two contributors. In the inner disk its formation is dependent on the gas and dust temperatures due to fast neutral-neutral reactions in the gas and potential sublimation of icy grains. Thus the $\mathrm{O} \rightarrow \mathrm{H}_{2} \mathrm{O}$ transition does not solely depend UV optical depth. Beyond a few AU and when the temperature falls below the water ice sublimation point, the fast gas-phase chemistry turns off and water is mostly as ice mediated by UV photodesorption. In the outer ( $>100 \mathrm{AU})$ disk photodesorption can also be important in the $\mathrm{CO}$ gas-ice transition when the temperature falls below its sublimation point and UV photons are present. The radial distances on the right ordinate are approximate and depend on the dust density profile and properties, the accretion rate, and the stellar spectral type.

$H \mathrm{I} \rightarrow \mathrm{H}_{2}$ : This transition is mediated by the impact of Ly $\alpha$ photons, which dominate the FUV radiation field and the reformation of $\mathrm{H}_{2}$ onto warm sub-micron sized dust grains in surface layers. A critical facet to understand for this layer, and for molecule formation in this transition zone in general, is the grain-surface formation of $\mathrm{H}_{2}$ at high dust temperatures (Cazaux and Tielens 2002, 2004, Cazaux et al. 2005).

$C$ II $\rightarrow C \rightarrow C O$ : Due to the high density of the inner disk $\left(\mathrm{n} \gg 10^{7} \mathrm{~cm}^{-3}\right)$ this allows for rapid build-up of a CO self-shielding column. Thus the $\mathrm{C}$ II $\rightarrow \mathrm{C} \rightarrow$ $\mathrm{CO}$ transition can be commensurate with that of $\mathrm{H}_{2}$ (Woitke et al. 2009; Najita et al. 2011). At larger distances from the star (many tens of AU), due to lower gas densities the carbon transition will shift away from $\mathrm{H}_{2}$ and become more similar to an interstellar PDR. In general, models use parametric descriptions of the CO photodissociation rate that incorporate shielding from $\mathrm{CO}, \mathrm{H}_{2}$, and dust (Visser et al. 2009). 
$\mathrm{O} \mathrm{I} \rightarrow \mathrm{OH} \rightarrow \mathrm{H}_{2} \mathrm{O}$ : Depending the strength of the UV field and gas heating, in the inner disk this transition occurs when $\mathrm{H}_{2}$ is present. In surface layers the dominant formation mechanism for water vapor is via fast neutral-neutral reactions that rapidly process all free $\mathrm{O}$ I into $\mathrm{H}_{2} \mathrm{O}$ when $\mathrm{T}_{\text {gas }}>400 \mathrm{~K}$ (Wagner and Graff 1987, Kaufman and Neufeld 1996). These reactions can become so fast that they can become competitive with photodestruction and $\mathrm{H}_{2} \mathrm{O}$ can self-shield (Bethell and Bergin 2009). Water has photoabsorption cross-sections that are continuous in the FUV (Yoshino et al. 1996); thus water can potentially shield other molecules from the destructive effects of UV in inner few AU (Bethell and Bergin 2009, Ádámkovics et al. 2014). Once the gas temperature falls below $400 \mathrm{~K}$ then the neutral-neutral gas phase pathways turn off. Formation then follows the less efficient ion-molecule pathways linked to $\mathrm{H}_{3} \mathrm{O}^{+}$and, in layers where $\mathrm{T}_{\text {dust }}<\mathrm{T}_{\text {sub }}\left(\mathrm{H}_{2} \mathrm{O}\right)$, requires UV photodesorption to release water and $\mathrm{OH}$ into the gas (Dominik et al. 2005, Hollenbach et al. 2009). The detailed review of the chemistry of water is presented in van Dishoeck et al. (2013a). Thus there will be a radial transition on the disk surface from hot to cold chemistry where the water abundance drops precipitously by orders of magnitude. The surface transition is nominally distinct from the midplane snowline.

$N$, $S$, and metal ions: It is expected that the $\mathrm{N}$ to $\mathrm{N}_{2}$ transition will be similar to that of $\mathrm{CO}$, at least in behavior, as $\mathrm{N}_{2}$ also can self-shield (Heays et al. 2014). The case of sulphur is more ambiguous as we have yet to locate the main repository of $\mathrm{S}$ in the dense interstellar medium and sulphur appears to mostly be missing from the gas (Druard and Wakelam|2012; Anderson et al.|2013). A similar statement can be made about heavy metal ions (e.g., Fe II, Si II, Mg II). In the dense ISM these heavy elements appear to be locked in the refractory solid state (Sofia et al. 1994, Maret and Bergin 2007) and the expectation is that this would also be the case in the protoplanetary disk. If these species were present in abundance they would be useful in mediating the interaction with the magnetic field (Perez-Becker and Chiang|2011; Ilgner and Nelson 2006).
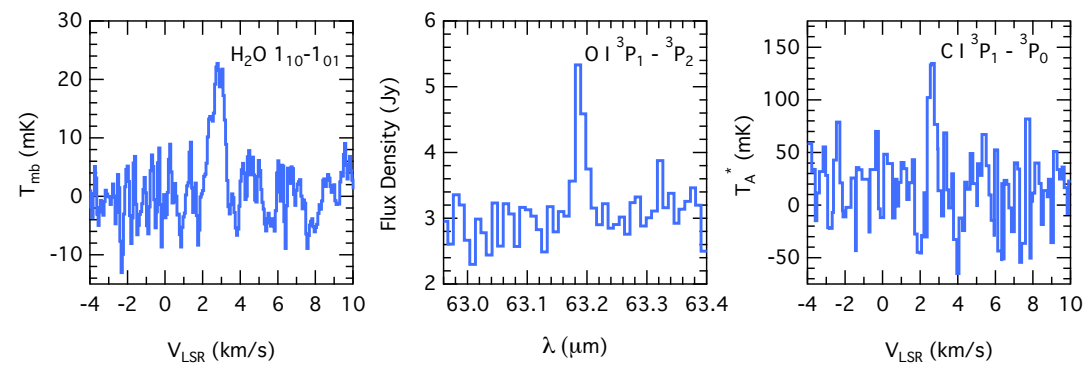

Fig. 11 Sample observations towards TW Hya of O II (Thi et al. 2010), C II (Kama et al. 2016), and $\mathrm{H}_{2} \mathrm{O}$ (Hogerheijde et al. 2011) ground state lines. Spectra obtained from Herschel Science Archive. 


\section{Observations}

For the $\mathrm{H}_{2}$ transition space telescopes such as FUSE and the Hubble Space Telescope can observe emission from the Lyman-Werner bands (Herczeg et al. 2002. France et al. 2012). Some of these emission lines coincide with the stellar Ly $\alpha$ emission and bear information on the true Ly $\alpha$ spectral profile impinging on the disk surface (Herczeg et al. 2002, France et al. 2014), which is important for models of chemistry (e.g., Fogel et al. 2011) and thermal balance (Ádámkovics et al. 2014).

Both C II and C I have emission lines that are observed via space-based platforms such as Herschel or via ground based observatories (for $\mathrm{CI}$ ). In general, the detection statistics for C II are quite poor (Howard et al. 2013), while C I (Fig. 11) has only been searched for in a handful of systems with moderate success (Tsukagoshi et al. 2015: Kama et al. 2016). Low-J CO lines are readily observed, but generically trace deeper layers within the disk surface.

For oxygen, O I has been surveyed with Herschel with a high rate of detection (e.g. Howard et al. 2013, and references therein). Analysis of the spectrally unresolved emission line shows that it is not clear whether the atomic oxygen emission traces the disk PDR or, in some sources, jets/outflows (Alonso-Martínez et al. 2017). $\mathrm{OH}$ and $\mathrm{H}_{2} \mathrm{O}$ have rotational transitions in the mid-infrared and vibrational modes in the near-IR that probe warm ( $\sim$ few hundred K) surface layers. Rotational line emission have been surveyed using Spitzer (Salyk et al. 2011; Pontoppidan et al. 2011) and vibrational lines via ground-based observatories (Salyk et al. 2008). Model analyses suggest that this emission is probing the PDR interior to the surface transition (Fig. 10 with a relatively high water abundance. Lower energy rotational lines emitting in the far-infrared probe gas at larger radii where material has temperatures well below the sublimation temperature of water ice. Thus, in this gas photodesorption is required to release water from grains( Herschel finds that detection rates are fairly low (below 10\%) with only two systems (TW Hya shown in Fig 11 and HD100546) having detectable emission lines (Du et al. 2017). This weak emission is interpreted as the result of a reduced water abundance in the photodesorbed gas (Hogerheijde et al. 2011; Du et al. 2017), but see also Kamp et al. (2013) for caveats regarding disk modeling uncertainties. Going forward, a combination of mid- and far-IR rotational emission can be used to constrain the surface water hot-cold transition (Zhang et al. 2013; Blevins et al. 2016). 


\section{Disk Composition and Planet Formation}

\section{Chemistry as a potentially controlling influence on planet formation}

The chemistry of the disk does far more than alter the composition of forming protoplanets, it can impact the formation process itself. As discussed in both the midplane and molecular layer theory, ice can impact the growth of grains by altering their sticking efficiency (e.g., Wang et al. 2005). Correspondingly, the presence of water ice may enhance the rate of dust growth, seeding the initial stages of planet formation. It should be noted that not all ice enhances sticking. Experiments of $\mathrm{CO}_{2}$ ice, for example, showed that $\mathrm{CO}_{2}$ and silicate grains had similar collisional behavior in terms of growth and fragmentation (Musiolik et al. 2016a b). Thus a fundamental understanding of the disk ice chemistry will be necessary for a complete treatment of dust evolution beyond $1 \mathrm{AU}$ in disks (e.g., Krijt et al.2016, Stammler et al.2017).

The physical properties of the gas, such as viscosity, temperature, turbulence, and ionization fraction, too can impact the growth rate of solids and the later stages of planet formation. The accretion rate of gas onto planets, along with how planets sculpt the disk itself, is related to the disk viscosity. This very viscosity can additional act to mix the disk gas, which can alter its chemical composition by bringing together material from vastly different regions in the disk. As discussed above, the viscosity is often parameterized with $\alpha$. Typical accretion rates onto the star require high values of $\alpha \sim 0.01$ (Hartmann et al. 1998). For low mass disks, the classical expectation is that magnetic coupling with the weakly ionized disk generates magnetically driven turbulence through the magneto-rotational instability (MRI; Balbus and Hawley 1991), which provides a source of disk viscosity.

There have been substantial efforts to measure the predicted non-Keplerian, nonthermal motions in disks, such as those expected to be introduced by MRI (Simon et al. 2015) using observations of the bulk molecular gas, both unresolved (Hughes et al. 2011) and resolved (Flaherty et al. 2015; Teague et al. 2016). So far, highly constraining limits have been placed (e.g., $<3 \%$ of the local sound speed in the HD163296 disk; Flaherty et al. 2015). Such estimates fundamentally depend on temperature, however, and thus caution must be taken when correcting out thermal motions versus turbulent motions (Teague et al.2016).

Nonetheless, such limits are an order of magnitude lower than MRI theory would predict, suggesting it is less efficient as a source of viscosity (and mixing) beyond 30 AU than previously thought. One possible explanation is that the disk may be more weakly ionized than expected, such that the gas and the magnetic fields are poorly coupled. Cleeves et al. (2015) used observations of molecular ions in the warm molecular layer to infer the distribution of ionizing sources, and the corresponding net ionization fraction of the TW Hya disk. The distribution of molecular ions was

found to be consistent with a low cosmic ray flux, and correspondingly, a low disk ionization fraction such that MRI should be inefficient for most of the disk midplane, out to $\sim 50-60 \mathrm{AU}$. 
This tension between $\alpha$ and accretion rates onto the star have another potential solution, namely to have the angular momentum carried away instead by magnetohydrodynamic disk winds (e.g. Konigl and Pudritz 2000; Salmeron et al. 2007, Suzuki and Inutsuka 2009, Béthune et al. 2017; Bai et al. 2016), built off of the earlier seminal work of Blandford and Payne (1982). However, these winds must not generate turbulence, especially in the upper layers where the observational constraints are most stringent (Simon et al. 2017). Using a series of shearing box simulations, Simon et al. (2017) investigated which parts of parameter space satisfy both high accretion and maintain a laminar disk state, and found that a net vertical magnetic field threading the disk is crucial to matching both conditions. Going forward, observational constraints on the disk magnetic field through, e.g., the Zeeman effect will help test this actively evolving theoretical picture.

\section{Global disk composition and its connection with gas giant planetary composition}

In general, the bulk of planetary gas giant atmospheres are in chemical equilibrium (except in specific instances, e.g.,photochemistry and transport induced quenching Moses 2014). Thus the particular molecular form of delivery for say carbon (e.g. $\mathrm{CH}_{4}, \mathrm{CO}, \mathrm{CO}_{2}$, hydrocarbons) will not matter; for a given pressure-temperature the expectation is that all carbon will reside in $\mathrm{CH}_{4}$ for $\operatorname{cool}(<1000 \mathrm{~K})$ atmospheres or $\mathrm{CO}$ for warmer conditions. Thus, if we seek to explore connections between giant planet atmospheric composition with the composition of the gas at formation we must look at elemental pools and target the main gas phase carriers of abundant elements.

The most successful connective tissue in this area relates to the $\mathrm{C} / \mathrm{O}$ ratio of the gas that changes radially within the disk (Öberg et al. 2011). Figure 12 illustrates how sublimation alone can change the ratio of carbon to oxygen in the ice versus gas in the midplane with stellar distance for a typical solar mass star. Beyond the $\mathrm{CO}$ snowline all the carbon and oxygen is frozen in the dust. Just inside the CO snowline the ratio will be near unity at $\mathrm{CO}$ carries the majority of gas phase $\mathrm{C}$ and $\mathrm{O}$ because water and $\mathrm{CO}_{2}$ remain frozen as ice (and so on).

In the core accretion picture, the radial position of an accreting protoplanet will determine the carbon to oxygen ratio in the core. For cores that are sufficiently massive to gravitationally capture a gas rich envelope, the carbon to oxygen ratio of the gas will tend to be the inverse of what has frozen out into solids. Since the main phase of gas accretion is rapid ( $\sim 10^{4}$ yrs; Chapter 6 by D'Angelo and Lissauer) the envelope $\mathrm{C} / \mathrm{O}$ ratio and elemental abundances will therefore reflect the local conditions at the birth site. This model is simplistic as it neglects additional sources or sink terms including, but not limited to pollution via planetesimal accretion (Pinhas et al. 2016) or mixing due to core ablation (Helled and Guillot 2017). However, it captures the basic underpinning of the process and motivates the need to determine $\mathrm{C} / \mathrm{O}$ ratios in both exoplanetary systems and in gas-rich disks. Similar 


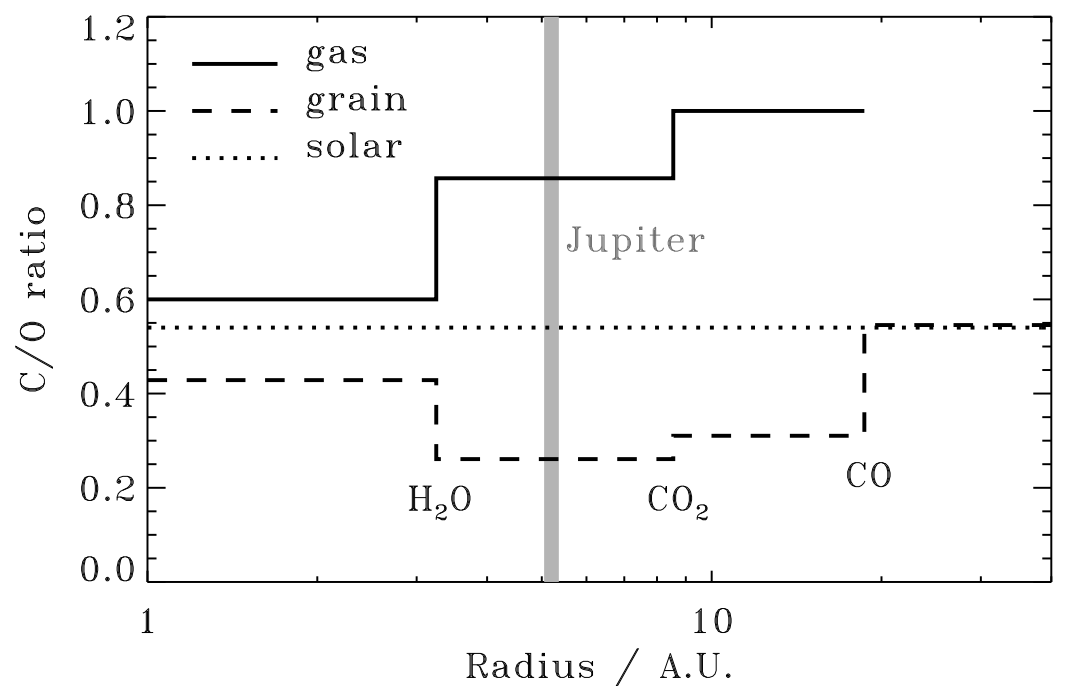

Fig. 12 The $\mathrm{C} / \mathrm{O}$ ratio in the gas and in grains, assuming the temperature structure of a typical protoplanetary disk around a solar-type star as given by Öberg et al. (2011). The $\mathrm{H}_{2} \mathrm{O}, \mathrm{CO}_{2}$, and $\mathrm{CO}$ snowline, along with the current location of Jupiter are marked for reference. Figure taken from Öberg et al. (2011).

explorations can be considered for nitrogen (Piso et al.|2016); however this must be tempered with the difficulty of detecting $\mathrm{N}_{2}$ (a main nitrogen carrier) in disks and in exoplanet atmospheres.

Of course, the disk is evolving as Fig. 12 presents a snapshot in time. Snowlines will move outwards during early high accretion stages and then move inwards during later stages as the accretion rate falls off and the gaseous disk dissipates (e.g., Hasegawa and Pudritz 2012, Cridland et al. 2016). These chemical effects will therefore be distributed radially as a function of the evolutionary state. Furthermore, the ice line itself might present a trap or a favorable site for planet formation which will leave its particular chemical fingerprint (Cridland et al. 2016). The full interplay is explored in the Chapters by Mordasini and Pudritz, respectively (this volume).

The other main area to explore connections relies on the overall elemental abundance relative to hydrogen. It is well known that Uranus and Neptune are enriched in ices; however, both Jupiter and Saturn exhibit enhanced abundances of carbon relative to solar (Flasar et al. 2005; Wong et al. 2008). In the case of Jupiter these enhancements are mirrored in numerous other elements and do not track solar abundances (Wong et al. 2008). The origin of these elemental abundance enhancements relative to solar is uncertain and has been attributed to pollution of the envelope via ice-rich planetesimals (Gautier et al. 2001), early formation during the phase of radial drift (Öberg and Bergin 2016), or perhaps dissipation of nebular hydrogen (Guillot and Hueso 2006). A key facet of this discussion is the disposition of oxygen in the Jovian planets which is unknown (Helled and Lunine 2014). For exoplanets, 
the question of abundance retrieval is complicated and can depend on the analysis but there are now suggestions in the literature for planets that have both superstellar and substellar abundances of C and O (e.g., Lavie et al. 2017, MacDonald and Madhusudhan 2017). To make connections to bulk abundances we need to trace key elemental carriers of $\mathrm{C}, \mathrm{O}$, and $\mathrm{N}$ within all phases of the disk lifetime while also having a reliable tracer of the hydrogen mass. The latter point is critical and we refer the reader to a recent review on this topic by Bergin and Williams (2017).

\section{Global disk composition and its connection with terrestrial planet composition}

For terrestrial worlds, in particular for the Earth, drawing connections between the chemical conditions at formation and their composition 4.6 Billion years later has a long history. As discussed above, water is a primary condensible in planetary systems. In the outer solar system Jovian moons and Kuiper Belt Objects often contain a significant amount of their mass as water ice (Grasset et al.|2017). In the case of the terrestrial planets, and the inner part of the asteroid belt, the water content is significantly less. The water mass fraction of the Earth has some uncertainty, but even extreme estimates are below 0.1\% of the Earth's mass (Mottl et al. 2007). Thus it is believed that the terrestrial worlds formed interior to the snowline.

A central question is then how the Earth, and by proxy other extrasolar terrestrial worlds that formed inside their respective water snowlines, received water content (see chapter by Izidoro and Raymond, this volume). This may also be related to the presence of other "volatile elements" such as carbon and nitrogen (and perhaps Sulphur) that are primarily contained in compounds with volatility comparable to or below that of water (e.g. Fig. 4). Put together there are implications for habitability writ large, and thus an important aspect is to use chemical tracers to probe the potential sources of origin for the volatile elements. Potential source terms include in situ formation via grains with tenuous amounts of water ice, delivery by icy planetesimals (comets) or rocky bodies that contain hydrous and carbonaceous minerals (asteroids), and nebular gas capture (van Dishoeck et al. 2013b). In this regard we can isolate several lines of inquiry that are major foci of observation, theoretical, and

laboratory work including: isotopic ratios and bulk composition. Another useful line of inquiry relates to noble gases which can be used to set constraints on delivery via comets (Marty et al. 2017).

\section{Isotopic Ratios and Chemical Fractionation}

Due to the Heisenberg Uncertainty Principle a molecule cannot exist at the bottom of the well that defines the potential of the interaction between the disparate atoms (e.g., Tennyson 2011). The minimum energy is called the zero point en- 
ergy, which is important for chemistry in cold regions. The ground state vibrational energy of the bond is $(1 / 2) h v_{B}$, where $v_{B}$ is the vibrational frequency of the bond. $v_{B}=(1 / 2 \pi) \sqrt{k / \mu}$ where $k$ is the force constant and $\mu$ is the reduced mass ( $\left.\mu=m_{1} \times m_{2} / m_{1}+m_{2}\right)$ with $m_{1}$ and $m_{1}$ the mass of the atoms (in the case for a two atom molecule). Because the force constant is invariant, this imprints a $1 / \sqrt{\mu}$ dependence on the zero-point energy between different isotopic variants, such as $\mathrm{H}_{2}$ and $\mathrm{HD},{ }^{12} \mathrm{CO}$ and ${ }^{13} \mathrm{CO}$. Thus, the bond is slightly stronger for the isotopically heavy molecule. This energy difference is small, but non-zero.

Of all the stable elements hydrogen has the largest mass/energy difference, with a value of $\sim 420 \mathrm{~K}$ (Irikura 2007). Chemical reactions that occur at temperatures well below this energy difference tend towards the stronger bond. Hence, for a reaction with $\mathrm{HD}$, the $\mathrm{D}$ will be preferentially transferred as opposed to the $\mathrm{H}$. This process is called fractionation and it can lead to very strong enhancements of the heavier isotope. In general isotopic enrichments of solar system material may provide a fingerprint that could provide clues regarding the formation environment of Earth's water and volatiles, but more broadly can hint at the origin of these same compounds in the disk or perhaps from the interstellar medium.

Hydrogen: The most well-studied isotope ratio pertains to the origins of the $\mathrm{D} / \mathrm{H}$ ratio of Earth's water. The measured value is given by Vienna Standard Mean Ocean Water (VSMOW) and is $\mathrm{D} / \mathrm{H}=1.5576 \times 10^{-4}$. The protosolar (hydrogen) value is estimated to be $\sim 2 \times 10^{-5}$ (Geiss and Gloeckler 2003) and thus Earth's water has excess deuterium. This facet is true of all measured water in interstellar bodies (Cleeves et al.|2014). The general trend, with some heterogeneity within certain classes of objects (meteorites, Kuiper belt comets), is that the outer solar system has slightly (factor of a few) higher $\mathrm{D} / \mathrm{H}$ ratios than water in the inner solar system. For a recent compilation of $\mathrm{D} / \mathrm{H}$ data see Altwegg et al. (2015).

The ultimate origin of the enhancement relates to this reaction and chemical kinetics (Millar et al. 1989):

$$
\mathrm{H}_{3}^{+}+\mathrm{HD} \leftrightarrow \mathrm{H}_{2} \mathrm{D}^{+}+\mathrm{H}_{2}+\Delta \mathrm{E},
$$

with $\Delta \mathrm{E}$ depending on whether the reactant is ortho- or para- $\mathrm{H}_{2}$. Below $\sim 30 \mathrm{~K}$, and in gas where the ortho/para ratio of $\mathrm{H}_{2}$ is low (Flower et al.2004) this reaction can produce orders of magnitude enrichments in deuterium containing molecules (e.g. $\mathrm{HDO}, \mathrm{CH}_{3} \mathrm{D}$, etc.). This requires a source of ionization (either cosmic-rays or $\mathrm{X}$ rays) to initiate. Thus, the high $\mathrm{D} / \mathrm{H}$ ratios cannot be created at $1 \mathrm{AU}$, as enriched water would rapidly re-equilibrate to the protosolar value (or slightly higher by a factor of $\sim 3$; Lécluse and Robert 1994). The source of high $\mathrm{D} / \mathrm{H}$ ratios is thus either the outer disk or the pre-stellar stage (Cleeves et al. 2014), and modern models explore the question of mixing inner and outer solar system material.

Oxygen and Nitrogen: The isotopes of oxygen have a much smaller mass difference when compared to hydrogen and kinetic chemical effects at low temperature are minimal (Langer and Graedel 1989). Thus the discovery of heavy oxygen isotopic enrichments in meteorites was a surprise (Clayton 1993). In equilibrium, which generally pertains to geochemical systems (such as the Earth or meteoritic progeni- 
tors), isotopic chemistry has a mass dependence such that the relative abundance of ${ }^{18} \mathrm{O} /{ }^{16} \mathrm{O}$ when plotted against ${ }^{17} \mathrm{O} /{ }^{16} \mathrm{O}$ has a slope of 2 . This reflects the mass difference between ${ }^{18} \mathrm{O}$ and ${ }^{17} \mathrm{O}$ relative to ${ }^{16} \mathrm{O}($ e.g. $18-16 / 17-16=2)$ and is consistent with isotopic ratios measured in Earth and Martian rocks. However, oxygen isotopes in meteoritic material, when plotted in this fashion, display a slope of unity and contain an excess of the heavier oxygen isotopes. This is not consistent with geochemistry and requires a different mechanism, which may provide clues to the origin of water in rocky bodies (Clayton et al. 1973, Clayton|1993, Thiemens 2006). A potential solution to these anomalies is photochemical self-shielding (Clayton 2002) which has a strong isotopic signature either in the disk (Lyons and Young 2005) or the parent cloud (Lee et al. 2008). Heavy nitrogen isotopes are also enriched in meteoritic and cometary material (Marty 2012). Here the potential origin may be linked to $\mathrm{N}_{2}$ self-shielding (Heays et al.2014) and/or kinetic isotopic effects (Charnley and Rodgers 2002).

\section{Astrochemical Foundations}

As we look towards a future where we have compositional information on terrestrial planet atmospheres beyond our own, we also must explore ways to understand how this composition might relate to conditions at formation. One feasible methodology is to explore bulk chemical composition in terms of the main carriers of $\mathrm{C}$ and $\mathrm{O}$ (N remains difficult to constrain). Water stands out as a prime tracer as it is the carrier of volatile oxygen (van Dishoeck et al.2013b) but there is potential to explore links in the carbon inventory as well (Bergin et al. 2015). However, there are clear challenges as terrestrial worlds likely have substantial sub-surface reservoirs and one must therefore draw concurrent links to mass/radius/density (e.g., Lopez and Fortney 2014).

At this moment we sit on the cusp of great discovery and our understanding of planet formation and its attendant effects on composition is rapidly expanding. Today with ALMA we are constraining the conditions that exist into the giant planet forming zone. With the widely anticipated launch of the James Webb Space Telescope and the new era of large telescopes the terrestrial planet forming zone will come into greater focus. At the same time, with the same instruments, we will be expanding our ability to characterize worlds from super-Earths, to mini-Neptunes, to Neptune-analogs, and finally massive gas giants such as Jupiter. Now is the time where the connective tissue of formation can be explored. First attempts at this summarized in chapters by Mordasini in particular, as well as Pudritz. In this light, the basic astrochemical foundation discussed here remains an essential tool to provide the interpretive framework to understand the origins of planet(esimal) composition. 


\section{References}

Ádámkovics M, Glassgold AE Najita JR (2014) Shielding by Water and OH in FUV and X-Ray Irradiated Protoplanetary Disks. ApJ786:135

Aikawa Y, van Zadelhoff GJ, van Dishoeck EF Herbst E (2002) Warm molecular layers in protoplanetary disks. A\&A386:622-632

Aikawa Y, Furuya K, Nomura H Qi C (2015) Analytical Formulae of Molecular Ion Abundances and the $\mathrm{N}_{2} \mathrm{H}^{+}$Ring in Protoplanetary Disks. ApJ807:120

Alonso-Martínez M, Riviere-Marichalar P, Meeus G et al. (2017) Herschel GASPS spectral observations of $\mathrm{T}$ Tauri stars in Taurus. Unraveling far-infrared line emission from jets and discs. A\&A603:A138

Altwegg K, Balsiger H, Bar-Nun A et al. (2015) 67P/Churyumov-Gerasimenko, a Jupiter family comet with a high D/H ratio. Science 347(27):1261952

Anderson DE, Bergin EA, Maret S Wakelam V (2013) New Constraints on the Sulfur Reservoir in the Dense Interstellar Medium Provided by Spitzer Observations of S I in Shocked Gas. ApJ779:141

Andrews SM, Wilner DJ, Espaillat C et al. (2011) Resolved Images of Large Cavities in Protoplanetary Transition Disks. ApJ732:42

Ardia P, Hirschmann MM, Withers AC Stanley BD (2013) Solubility of $\mathrm{CH}_{4}$ in a synthetic basaltic melt, with applications to atmosphere-magma ocean-core partitioning of volatiles. Geochim Cosmochim Acta114:52-71

Bai XN, Ye J, Goodman J Yuan F (2016) Magneto-thermal Disk Winds from Protoplanetary Disks. ApJ818:152

Balbus SA Hawley JF (1991) A powerful local shear instability in weakly magnetized disks. I Linear analysis. II - Nonlinear evolution. ApJ376:214-233

Bergin E, Calvet N, Sitko ML et al. (2004) A New Probe of the Planet-forming Region in T Tauri Disks. ApJ614:L133-L136

Bergin EA Williams JP (2017) The Determination of Protoplanetary Disk Masses. Formation, Evolution, and Dynamics of Young Solar Systems 445:in press

Bergin EA, Alves J, Huard T Lada CJ (2002) $\mathrm{N}_{2} \mathrm{H}^{+}$and $\mathrm{C}^{18}$ O Depletion in a Cold Dark Cloud. ApJ570:L101-L104

Bergin EA, Aikawa Y, Blake GA van Dishoeck EF (2007) The Chemical Evolution of Protoplanetary Disks. In: Protostars and Planets V, p 751

Bergin EA, Cleeves LI, Crockett N Blake GA (2014) Exploring the origins of carbon in terrestrial worlds[dagger]. Faraday Discuss 168:61-79, URL http://dx.doi.org/10.1039/ C4FD00003J

Bergin EA, Blake GA, Ciesla F, Hirschmann MM Li J (2015) Tracing the ingredients for a habitable earth from interstellar space through planet formation. Proceedings of the National Academy of Science 112:8965-8970

Bergin EA, Du F, Cleeves LI et al. (2016) Hydrocarbon Emission Rings in Protoplanetary Disks Induced by Dust Evolution. ApJ831:101

Bethell T Bergin E (2009) Formation and Survival of Water Vapor in the Terrestrial Planet-Forming Region. Science 326:1675-

Bethell TJ Bergin EA (2011a) Photoelectric Cross-sections of Gas and Dust in Protoplanetary Disks. ApJ740:7

Bethell TJ Bergin EA (2011b) The Propagation of Ly $\alpha$ in Evolving Protoplanetary Disks. ApJ739:78

Béthune W, Lesur G Ferreira J (2017) Global simulations of protoplanetary disks with net magnetic flux. I. Non-ideal MHD case. A\&A600:A75

Blandford RD Payne DG (1982) Hydromagnetic flows from accretion discs and the production of radio jets. MNRAS199:883-903

Blevins SM, Pontoppidan KM, Banzatti A et al. (2016) Measurements of water surface snow lines in classical protoplanetary disks. ApJp in press. 
Calvet N, Patino A, Magris GC D'Alessio P (1991) Irradiation of accretion disks around young objects. I - Near-infrared CO bands. ApJ380:617-630

Cazaux S Tielens AGGM (2002) Molecular Hydrogen Formation in the Interstellar Medium. ApJ575:L29-L32

Cazaux S Tielens AGGM (2004) $\mathrm{H}_{2}$ Formation on Grain Surfaces. ApJ604:222-237

Cazaux S, Caselli P, Tielens AGGM, LeBourlot J Walmsley M (2005) Molecular Hydrogen formation on grain surfaces. In: Saija R Cecchi-Pestellini C (eds) Journal of Physics Conference Series, Journal of Physics Conference Series, vol 6, pp 155-160, DOI 10.1088/1742-6596/6/1/016

Charnley SB (1997) Chemical models of interstellar gas-grain processes. III - Molecular depletion in NGC 2024. MNRAS291:455

Charnley SB Rodgers SD (2002) The End of Interstellar Chemistry as the Origin of Nitrogen in Comets and Meteorites. ApJ569:L133-L137

Chiang EI Goldreich P (1997) Spectral Energy Distributions of T Tauri Stars with Passive Circumstellar Disks. ApJ490:368-+

Clayton RN (1993) Oxygen isotopes in meteorites. Annual Review of Earth and Planetary Sciences 21:115-149

Clayton RN (2002) Solar System: Self-shielding in the solar nebula. Nature415:860-861

Clayton RN, Grossman L Mayeda TK (1973) A Component of Primitive Nuclear Composition in Carbonaceous Meteorites. Science 182:485-488

Cleeves LI (2016) Multiple Carbon Monoxide Snow Lines in Disks Sculpted by Radial Drift. ApJ816:L21

Cleeves LI, Adams FC Bergin EA (2013) Exclusion of Cosmic Rays in Protoplanetary Disks: Stellar and Magnetic Effects. ApJ772:5

Cleeves LI, Bergin EA, Alexander CMO et al. (2014) The ancient heritage of water ice in the solar system. Science 345:1590-1593

Cleeves LI, Bergin EA, Qi C, Adams FC Öberg KI (2015) Constraining the X-Ray and CosmicRay Ionization Chemistry of the TW Hya Protoplanetary Disk: Evidence for a Sub-interstellar Cosmic-Ray Rate. ApJ799:204

Cridland AJ, Pudritz RE Birnstiel T (2016) Radial Drift of Dust in Protoplanetary Disks: The Evolution of Ice lines and Dead zones. MNRAS p submitted

D’Alessio P, Calvet N, Hartmann L, Lizano S Cantó J (1999) Accretion Disks around Young Objects. II. Tests of Well-mixed Models with ISM Dust. ApJ527:893-909

D'Alessio P, Calvet N Hartmann L (2001) Accretion Disks around Young Objects. III. Grain Growth. ApJ553:321-334

D'Alessio P, Calvet N Woolum DS (2005) Thermal Structure of Protoplanetary Disks. In: Krot AN, Scott ERD Reipurth B (eds) Chondrites and the Protoplanetary Disk, Astronomical Society of the Pacific Conference Series, vol 341, pp 353-+

D’Alessio P, Calvet N, Hartmann L, Franco-Hernández R Servín H (2006) Effects of Dust Growth and Settling in T Tauri Disks. ApJ638:314-335

Dalgarno A (2006) Interstellar Chemistry Special Feature: The galactic cosmic ray ionization rate. Proceedings of the National Academy of Science 103:12,269-12,273

Debes JH, Jang-Condell H, Weinberger AJ, Roberge A Schneider G (2013) The 0.5-2.22 $\mu \mathrm{m}$ Scattered Light Spectrum of the Disk around TW Hya: Detection of a Partially Filled Disk Gap at $80 \mathrm{AU}$. ApJ771:45

Dominik C, Ceccarelli C, Hollenbach D Kaufman M (2005) Gas-Phase Water in the Surface Layer of Protoplanetary Disks. ApJ635:L85-L88

Druard C Wakelam V (2012) Polysulphanes on interstellar grains as a possible reservoir of interstellar sulphur. MNRAS426:354-359

Du F Bergin EA (2014) Water Vapor Distribution in Protoplanetary Disks. ApJ792:2

Du F, Bergin EA Hogerheijde MR (2015) Volatile depletion in the TW Hydrae disk atmosphere. ApJ807:L32

Du F, Bergin EA, Hogerheijde M et al. (2017) Survey of Cold Water Lines in Protoplanetary Disks: Indications of Systematic Volatile Depletion. ApJ842:98 
Dullemond CP Dominik C (2004) The effect of dust settling on the appearance of protoplanetary disks. A\&A421:1075-1086

Dutrey A, Semenov D, Chapillon E et al. (2014) Physical and Chemical Structure of PlanetForming Disks Probed by Millimeter Observations and Modeling. Protostars and Planets VI pp 317-338

Dutrey A, Guilloteau S, Piétu V et al. (2017) The Flying Saucer: Tomography of the thermal and density gas structure of an edge-on protoplanetary disk. ArXiv e-prints

Facchini S, Birnstiel T, Bruderer S van Dishoeck EF (2017) Different dust and gas radial extents in protoplanetary disks: consistent models of grain growth and CO emission. A\&A605:A16

Fayolle EC, Balfe J, Loomis R et al. (2016) $\mathrm{N}_{2}$ and CO Desorption Energies from Water Ice. ApJ816:L28

Flaherty KM, Hughes AM, Rosenfeld KA et al. (2015) Weak Turbulence in the HD 163296 Protoplanetary Disk Revealed by ALMA CO Observations. ApJ813:99

Flasar FM, Achterberg RK, Conrath BJ et al. (2005) Titan's Atmospheric Temperatures, Winds, and Composition. Science 308:975-978

Flower DR, Pineau des Forêts G Walmsley CM (2004) Multiply-deuterated species in prestellar cores. A\&A427:887-893

Fogel JKJ, Bethell TJ, Bergin EA, Calvet N Semenov D (2011) Chemistry of a Protoplanetary Disk with Grain Settling and Ly $\alpha$ Radiation. ApJ726:29

France K, Schindhelm E, Herczeg GJ et al. (2012) A Hubble Space Telescope Survey of $\mathrm{H}_{2}$ Emission in the Circumstellar Environments of Young Stars. ApJ756:171

France K, Herczeg GJ, McJunkin M Penton SV (2014) $\mathrm{CO} / \mathrm{H}_{2}$ Abundance Ratio $\sim 10^{-4}$ in a Protoplanetary Disk. ApJ794:160

Fraser HJ, Collings MP, McCoustra MRS Williams DA (2001) Thermal desorption of water ice in the interstellar medium. MNRAS327:1165-1172

Furlan E et al. (2005) Colors of Classical T Tauri Stars in Taurus Derived from Spitzer Infrared Spectrograph Spectra: Indication of Dust Settling. ApJ628:L65-L68

Furuya K Aikawa Y (2014) Reprocessing of Ices in Turbulent Protoplanetary Disks: Carbon and Nitrogen Chemistry. ApJ790:97

Gautier D, Hersant F, Mousis O Lunine JI (2001) Enrichments in Volatiles in Jupiter: A New Interpretation of the Galileo Measurements. ApJ550:L227-L230

Geiss J Gloeckler G (2003) Isotopic Composition of H, HE and NE in the Protosolar Cloud. Space Sci Rev106:3-18

Gorti U Hollenbach D (2004) Models of Chemistry, Thermal Balance, and Infrared Spectra from Intermediate-Aged Disks around G and K Stars. ApJ613:424-447

Grasset O, Castillo-Rogez J, Guillot T, Fletcher LN Tosi F (2017) Water and Volatiles in the Outer Solar System. Space Sci Rev

Güdel M (2004) X-ray astronomy of stellar coronae. A\&A Rev12:71-237

Guillot T Hueso R (2006) The composition of Jupiter: sign of a (relatively) late formation in a chemically evolved protosolar disc. MNRAS367:L47-L51

Gullbring E, Calvet N, Muzerolle J Hartmann L (2000) The Structure and Emission of the Accretion Shock in T Tauri Stars. II. The Ultraviolet-Continuum Emission. ApJ544:927-932

Habing HJ (1968) The interstellar radiation density between $912 \mathrm{~A}$ and $2400 \mathrm{~A}$. Bull Astron Inst Netherlands19:421-+

Hartmann L, Calvet N, Gullbring E D'Alessio P (1998) Accretion and the Evolution of T Tauri Disks. ApJ495:385-400

Hasegawa Y Pudritz RE (2012) Evolutionary Tracks of Trapped, Accreting Protoplanets: The Origin of the Observed Mass-Period Relation. ApJ760:117

Hayashi C (1981) Structure of the Solar Nebula, Growth and Decay of Magnetic Fields and Effects of Magnetic and Turbulent Viscosities on the Nebula. Progress of Theoretical Physics Supplement 70:35-53

Heays AN, Visser R, Gredel R et al. (2014) Isotope selective photodissociation of $\mathrm{N}_{2}$ by the interstellar radiation field and cosmic rays. A\&A562:A61 
Helled R Guillot T (2017) Internal Structure of Giant and Icy Planets: Importance of Heavy Elements and Mixing. ArXiv e-prints

Helled R Lunine J (2014) Measuring Jupiter's water abundance by Juno: the link between interior and formation models. MNRAS441:2273-2279

Henke BL, Gullikson EM Davis JC (1993) X-Ray Interactions: Photoabsorption, Scattering, Transmission, and Reflection at $\mathrm{E}=50-30,000 \mathrm{eV}, \mathrm{Z}=1-92$. Atomic Data and Nuclear Data Tables 54:181-342

Henning T Semenov D (2013) Chemistry in Protoplanetary Disks. ArXiv e-prints

Herbst E Klemperer W (1973) The Formation and Depletion of Molecules in Dense Interstellar Clouds. ApJ185:505-534

Herczeg GJ, Linsky JL, Valenti JA, Johns-Krull CM Wood BE (2002) The Far-Ultraviolet Spectrum of TW Hydrae. I. Observations of $\mathrm{H}_{2}$ Fluorescence. ApJ572:310-325

Herrero VJ, Gálvez Ó, Maté B Escribano R (2010) Interaction of CH4 and H2O in ice mixtures. Physical Chemistry Chemical Physics (Incorporating Faraday Transactions) 12:3164

Hogerheijde MR, Bergin EA, Brinch C et al. (2011) Detection of the Water Reservoir in a Forming Planetary System. Science 334:338-340

Hollenbach D, Kaufman MJ, Bergin EA Melnick GJ (2009) Water, $\mathrm{O}_{2}$, and Ice in Molecular Clouds. ApJ690:1497-1521

Hollenbach DJ Tielens AGGM (1999) Photodissociation regions in the interstellar medium of galaxies. Reviews of Modern Physics 71:173-230

Howard CD, Sandell G, Vacca WD et al. (2013) Herschel/PACS Survey of Protoplanetary Disks in Taurus/Auriga Observations of [O I] and [C II], and Far-infrared Continuum. ApJ776:21

Hughes AM, Wilner DJ, Andrews SM, Qi C Hogerheijde MR (2011) Empirical Constraints on Turbulence in Protoplanetary Accretion Disks. ApJ727:85

Igea J Glassgold AE (1999) X-Ray Ionization of the Disks of Young Stellar Objects. ApJ518:848858

Ilgner M Nelson RP (2006) On the ionisation fraction in protoplanetary disks. II. The effect of turbulent mixing on gas-phase chemistry. A\&A445:223-232

Irikura KK (2007) Experimental Vibrational Zero-Point Energies: Diatomic Molecules. Journal of Physical and Chemical Reference Data 36:389-397

Isella A, Carpenter JM Sargent AI (2009) Structure and Evolution of Pre-main-sequence Circumstellar Disks. ApJ701:260-282

Kama M, Bruderer S, van Dishoeck EF et al. (2016) Volatile carbon locking and release in protoplanetary disks. A study of TW Hya and HD 100546. A\&Ap in press.

Kamp I Dullemond CP (2004) The Gas Temperature in the Surface Layers of Protoplanetary Disks. ApJ615:991-999

Kamp I, Thi WF, Meeus G et al. (2013) Uncertainties in water chemistry in disks: An application to TW Hydrae. A\&A559:A24

Kastner JH, Qi C, Gorti U et al. (2015) A Ring of $\mathrm{C}_{2} \mathrm{H}$ in the Molecular Disk Orbiting TW Hya. ApJ806:75

Kaufman MJ Neufeld DA (1996) Water Maser Emission from Magnetohydrodynamic Shock Waves. ApJ456:250-+

Kenyon SJ Hartmann L (1995) Pre-Main-Sequence Evolution in the Taurus-Auriga Molecular Cloud. ApJS101:117-+

Konigl A Pudritz RE (2000) Disk Winds and the Accretion-Outflow Connection. Protostars and Planets IV $\mathrm{p} 759$

Krijt S, Ciesla FJ Bergin EA (2016) Tracing Water Vapor and Ice During Dust Growth. ApJp in press

Langer WD Graedel TE (1989) Ion-molecule chemistry of dense interstellar clouds - Nitrogen-, oxygen-, and carbon-bearing molecule abundances and isotopic ratios. ApJS69:241-269

Lavie B, Mendonça JM, Mordasini C et al. (2017) HELIOS-RETRIEVAL: An Open-source, Nested Sampling Atmospheric Retrieval Code; Application to the HR 8799 Exoplanets and Inferred Constraints for Planet Formation. AJ154:91 
Lécluse C Robert F (1994) Hydrogen isotope exchange reaction rates: Origin of water in the inner solar system. Geochim Cosmochim Acta58:2927-2939

Lee JE, Bergin EA Lyons JR (2008) Oxygen isotope anomalies of the Sun and the original environment of the solar system. Meteoritics and Planetary Science 43:1351-1362

Loomis RA, Cleeves LI, Öberg KI, Guzman VV Andrews SM (2015) The Distribution and Chemistry of $\mathrm{H}_{2} \mathrm{CO}$ in the DM Tau Protoplanetary Disk. ApJ809:L25

Lopez ED Fortney JJ (2014) Understanding the Mass-Radius Relation for Sub-neptunes: Radius as a Proxy for Composition. ApJ792:1

Lyons JR Young ED (2005) CO self-shielding as the origin of oxygen isotope anomalies in the early solar nebula. Nature435:317-320

MacDonald RJ Madhusudhan N (2017) HD 209458b in new light: evidence of nitrogen chemistry, patchy clouds and sub-solar water. MNRAS469:1979-1996

Maret S Bergin EA (2007) The Ionization Fraction of Barnard 68: Implications for Star and Planet Formation. ApJ664:956

Martín-Doménech R, Muñoz Caro GM, Bueno J Goesmann F (2014) Thermal desorption of circumstellar and cometary ice analogs. A\&A564:A8

Marty B (2012) The origins and concentrations of water, carbon, nitrogen and noble gases on Earth. Earth and Planetary Science Letters 313:56-66

Marty B, Altwegg K, Balsiger H et al. (2017) Xenon isotopes in 67P/Churyumov-Gerasimenko show that comets contributed to Earth's atmosphere. Science 356:1069-1072

Millar TJ, Bennett A Herbst E (1989) Deuterium fractionation in dense interstellar clouds. ApJ340:906-920

Morrison R McCammon D (1983) Interstellar photoelectric absorption cross sections, $0.03-10 \mathrm{keV}$. ApJ270:119-122

Moses JI (2014) Chemical kinetics on extrasolar planets. Philosophical Transactions of the Royal Society of London Series A 372:20130,073-20130,073

Mottl M, Glazer B, Kaiser R Meech K (2007) Water and astrobiology. Chemie der Erde / Geochemistry 67:253-282

Musiolik G, Teiser J, Jankowski T Wurm G (2016a) Collisions of $\mathrm{CO}_{2}$ Ice Grains in Planet Formation. ApJ818:16

Musiolik G, Teiser J, Jankowski T Wurm G (2016b) Ice Grain Collisions in Comparison: CO2, $\mathrm{H} 2 \mathrm{O}$, and Their Mixtures. ApJ827:63

Najita JR, Ádámkovics M Glassgold AE (2011) Formation of Organic Molecules and Water in Warm Disk Atmospheres. ApJ743:147

Nomura H Millar TJ (2005) Molecular hydrogen emission from protoplanetary disks. A\&A438:923-938

Öberg KI Bergin EA (2016) Excess C/O and C/H in Outer Protoplanetary Disk Gas. ApJ831:L19

Öberg KI, van Dishoeck EF Linnartz H (2009) Photodesorption of ices I: $\mathrm{CO}, \mathrm{N}_{2}$, and $\mathrm{CO}_{2}$. A\&A496:281-293

Öberg KI, Murray-Clay R Bergin EA (2011) The Effects of Snowlines on C/O in Planetary Atmospheres. ApJ743:L16

Öberg KI, Furuya K, Loomis R et al. (2015) Double $\mathrm{DCO}^{+}$Rings Reveal CO Ice Desorption in the Outer Disk Around IM Lup. ApJ810:112

Padovani M, Galli D Glassgold AE (2009) Cosmic-ray ionization of molecular clouds. A\&A501:619-631

Perez-Becker D Chiang E (2011) Surface Layer Accretion in Conventional and Transitional Disks Driven by Far-ultraviolet Ionization. ApJ735:8

Pinhas A, Madhusudhan N Clarke C (2016) Efficiency of planetesimal ablation in giant planetary envelopes. MNRAS463:4516-4532

Pinte C, Dent WRF, Ménard F et al. (2016) Dust and Gas in the Disk of HL Tauri: Surface Density, Dust Settling, and Dust-to-gas Ratio. ApJ816:25

Piso AMA, Pegues J Öberg KI (2016) The Role of Ice Compositions for Snowlines and the C/N/O Ratios in Active Disks. ApJ833:203 
Pontoppidan KM, Blake GA Smette A (2011) The Structure and Dynamics of Molecular Gas in Planet-forming Zones: A CRIRES Spectro-astrometric Survey. ApJ733:84

Preibisch T, Kim YC, Favata F et al. (2005) The Origin of T Tauri X-Ray Emission: New Insights from the Chandra Orion Ultradeep Project. ApJS160:401-422

Qi C, Oberg KI, Wilner DJ et al. (2013) Imaging of the CO Snow Line in a Solar Nebula Analog. Science 341:630

Qi C, Öberg KI, Andrews SM et al. (2015) Chemical Imaging of the CO Snow Line in the HD 163296 Disk. ApJ813:128

Reboussin L, Wakelam V, Guilloteau S, Hersant F Dutrey A (2015) Chemistry in protoplanetary disks: the gas-phase $\mathrm{CO} / \mathrm{H}_{2}$ ratio and the carbon reservoir. A\&A579:A82

Salmeron R, Königl A Wardle M (2007) Angular momentum transport in protostellar discs. MNRAS375:177-183

Salyk C, Pontoppidan KM, Blake GA et al. (2008) $\mathrm{H}_{2} \mathrm{O}$ and $\mathrm{OH}$ Gas in the Terrestrial Planetforming Zones of Protoplanetary Disks. ApJ676:L49-L52

Salyk C, Pontoppidan KM, Blake GA, Najita JR Carr JS (2011) A Spitzer Survey of Mid-infrared Molecular Emission from Protoplanetary Disks. II. Correlations and Local Thermal Equilibrium Models. ApJ731:130

Schindhelm E, France K, Herczeg GJ et al. (2012) Ly $\alpha$ Dominance of the Classical T Tauri Farultraviolet Radiation Field. ApJ756:L23

Schwarz KR, Bergin EA, Cleeves LI et al. (2016) The Radial Distribution of $\mathrm{H}_{2}$ and CO in TW Hya as Revealed by Resolved ALMA Observations of CO Isotopologues. ApJ823:91

Shakura NI Syunyaev RA (1973) Black holes in binary systems. Observational appearance. A\&A24:337-355

Simon JB, Hughes AM, Flaherty KM, Bai XN Armitage PJ (2015) Signatures of MRI-driven Turbulence in Protoplanetary Disks: Predictions for ALMA Observations. ApJ808:180

Simon JB, Bai XN, Flaherty KM Hughes AM (2017) A New Model for Weak Turbulence in Protoplanetary Disks. ArXiv e-prints

Sofia UJ, Cardelli JA Savage BD (1994) The abundant elements in interstellar dust. ApJ430:650 666

Stammler SM, Birnstiel T, Panić O, Dullemond CP Dominik C (2017) Redistribution of CO at the location of the CO ice line in evolving gas and dust disks. A\&A600:A140

Stevenson DJ Lunine JI (1988) Rapid formation of Jupiter by diffuse redistribution of water vapor in the solar nebula. Icarus75:146-155

Suzuki TK Inutsuka Si (2009) Disk Winds Driven by Magnetorotational Instability and Dispersal of Protoplanetary Disks. ApJ691:L49-L54

Teague R, Guilloteau S, Semenov D et al. (2016) Measuring turbulence in TW Hydrae with ALMA: methods and limitations. A\&A592:A49

Tennyson J (2011) Astronomical Spectroscopy: AN Introduction to the Atomic and Molecular Physics of Astronomical Spectra (2ND Edition). World Scientific, DOI 10.1142/7574

Thi WF, Mathews G, Ménard F et al. (2010) Herschel-PACS observation of the 10 Myr old T Tauri disk TW Hya. Constraining the disk gas mass. A\&A518:L125

Thiemens MH (2006) History and Applications of Mass-Independent Isotope Effectsa. Annual Review of Earth and Planetary Sciences 34:217-262

Tsukagoshi T, Momose M, Saito M et al. (2015) First Detection of [C I] ${ }^{3} \mathrm{P}_{1}-{ }^{3} \mathrm{P}_{0}$ Emission from a Protoplanetary Disk. ApJ802:L7

Umebayashi T Nakano T (1981) Fluxes of Energetic Particles and the Ionization Rate in Very Dense Interstellar Clouds. PASJ33:617

van Dishoeck EF, Herbst E Neufeld DA (2013a) Interstellar Water Chemistry: From Laboratory to Observations. Chemical Reviews 113:9043-9085

van Dishoeck EF, Herbst E Neufeld DA (2013b) Interstellar Water Chemistry: From Laboratory to Observations. Chemical Reviews 113:9043-9085

van Zadelhoff GJ, van Dishoeck EF, Thi WF Blake GA (2001) Submillimeter lines from circumstellar disks around pre-main sequence stars. A\&A377:566-580 
van't Hoff MLR, Walsh C, Kama M, Facchini S van Dishoeck EF (2017) Robustness of $\mathrm{N}_{2} \mathrm{H}^{+}$as tracer of the CO snowline. A\&A599:A101

Visser R, van Dishoeck EF Black JH (2009) The photodissociation and chemistry of CO isotopologues: applications to interstellar clouds and circumstellar disks. A\&A503:323-343

Wagner AF Graff MM (1987) Oxygen chemistry of shocked interstellar clouds. I - Rate constants for thermal and nonthermal internal energy distributions. ApJ317:423-431

Wang H, Bell RC, Iedema MJ, Tsekouras AA Cowin JP (2005) Sticky Ice Grains Aid Planet Formation: Unusual Properties of Cryogenic Water Ice. ApJ620:1027-1032

Webber WR (1998) A New Estimate of the Local Interstellar Energy Density and Ionization Rate of Galactic Cosmic Cosmic Rays. ApJ506:329-334

Weidenschilling SJ (1977) The distribution of mass in the planetary system and solar nebula. Ap\&SS51:153-158

Weidenschilling SJ Cuzzi JN (1993) Formation of planetesimals in the solar nebula. In: Levy EH Lunine JI (eds) Protostars and Planets III, pp 1031-1060

Whipple FL (1973) Radial Pressure in the Solar Nebula as Affecting the Motions of Planetesimals. NASA Special Publication 319:355

Woitke P, Kamp I Thi WF (2009) Radiation thermo-chemical models of protoplanetary disks. I. Hydrostatic disk structure and inner rim. A\&A501:383-406

Wong MH, Lunine JI, Atreya S et al. (2008) conference two. in Reviews in Mineralogy and Geochemistry: Oxygen in the Earliest Solar System, (eds G McPherson et al), Mineralogical Society of America, 68, 219

Yang H, Herczeg GJ, Linsky JL et al. (2012) A Far-ultraviolet Atlas of Low-resolution Hubble Space Telescope Spectra of T Tauri Stars. ApJ744:121

Yoshino K, Esmond JR, Parkinson WH, Ito K Matsui T (1996) Absorption Cross Section Measurements of Water Vapor in the Wavelength Region $120 \mathrm{~nm}$ to $188 \mathrm{~nm}$. Chemical Physics 211:387-+

Zhang K, Pontoppidan KM, Salyk C Blake GA (2013) Evidence for a Snow Line beyond the Transitional Radius in the TW Hya Protoplanetary Disk. ApJ766:82

Zhang K, Bergin EA, Blake GA, Cleeves L Schwarz K (2017) Unvieling the mass inventory of the giant-planet formation zone in a solar nebula analog. Nature Astronomy p submitted 